\title{
Modeling and Simulation of the Effects of Social Relation and Emotion on Decision Making in Emergency Evacuation
}

\author{
Xuan Hien $\mathrm{Ta}^{1}$ \\ ${ }^{1}$ Toulouse University, UPS-IRIT, Toulouse, France \\ Dominique Longin ${ }^{1}$ \\ ${ }^{1}$ Toulouse University, CNRS-IRIT, Toulouse, France
}

\author{
Manh Hung Nguyen ${ }^{3,4}$ \\ ${ }^{3}$ Posts and Telecommunications Institute of Technology (PTIT) \\ ${ }^{4}$ UMI UMMISCO 209 (IRD/UPMC), Hanoi, Vietnam
}

\author{
Benoit Gaudou ${ }^{1}$ \\ ${ }^{1}$ Toulouse University, UT1C-IRIT, Toulouse, France \\ Tuong Vinh $\mathrm{Ho}^{2,4}$ \\ ${ }^{2}$ IFI, Vietnam National University in Hanoi \\ ${ }^{4}$ UMI UMMISCO 209 (IRD/UPMC), Hanoi, Vietnam
}

\begin{abstract}
Applying agent-based modeling to simulate the evacuation in case of emergency situations is recognized by many research works as an efficient tool for understanding the behavior and decision making of occupants in these situations. In this paper, we present our work aiming to modeling the influence of the emotion and social relationship of occupants on their behaviors and decision making in emergency as in case of fire disaster. Firstly, we proposed a formalization of occupants' behavior at group level in emergency situations based on the social theory. This formalization details possible behaviors and actions of people in emergency evacuations, taking into account occupant's social relationship. The formalization will facilitate the construction of simulation for emergency evacuation. Secondly, we modeled the influence of emotion and group behavior on the decision making of occupants in crisis situations. Thirdly, we developed an agent-based simulation that took into account the effect of group and emotion on the decision making of occupants in emergency situations. We conducted a set of experiments allowing to observe and analyze the behavior of people in emergency evacuation.
\end{abstract}

Keywords-Agent-based simulation; emotion; social relation; emergency evacuation

\section{INTRODUCTION}

Fire disaster is one of the terrible causes of casualties in today's life. It is often reported that casualties are primarily caused by poor decision-making during a fire disaster. Since many years, applying agent-based modeling (ABM) to simulate the occupant's evacuation in buildings in case of fire or emergency situations has been recognized by many research works as an efficient tool for understanding the behavior and decision making of occupants in these situations. Making a fire evacuation simulation as realistic as possible is a complex task that requires to taking into account many aspects such as: the environment (the building for example), the behavior of occupants, the creation and propagation of fires and smokes, the decision-making process, and more. In our previous works, we have gradually taken certain levels of these aspects in the modeling and developing fire evacuation simulation. The model presented in [1] not only simulates the effects of fire/smoke on the abilities to move, to observe evacuees, but also takes into account the given advice of fire evacuation experts. [2] presents a new model of emotional contagion based on some main findings in social psychology that allows to studying the emotion dynamics at individual and group levels in emergency situation. This paper presents a modeling of the influence of the emotion and social relationship of occupants on their behaviors and decision making in emergency as in case of fire disaster. The paper contributions are: 1) a formalization of occupants' behavior at group level in emergency situations based on the social theory, taking into account social relations; 2) the modeling of the influence of emotion and group behavior on the decision making of occupants in crisis situations; and 3) an agent-based fire evacuation simulation that took into account the effects of group and emotion on the decision making of occupants in emergency situations.

This paper is organized as follows: Section II presents some related works in the field of crowd evacuation modeling and simulation in emergency situations, focusing on emotion, group behavior and decision-making process. Section III presents our proposed formalization for modeling group behavior in case of emergency, taking into account social relations. Section IV presents the modeling of the influence of emotion and group behavior on decision-making process of group. Section V describes the implemented simulation and experimental results. Finally, Section VI presents some conclusions as well as a discussion about future research.

\section{RELATED WORKS}

Regarding agent-based simulation for fire evacuation in buildings, [1] provided a summary of a partial collection of research works in this field. However, most of the works in this summary did not explicitly deal with the modeling of group behavior and the influence of emotion on decision-making process in emergency situations. 


\section{A. Behaviours in Crisis Situation}

We discuss hereafter some works related to group behavior in emergency situations. On the simulation side, the social interaction was taken into account in several simulations [3][5]. [3] introduces the intra-group (related to relationship of members inside the group) effect and inter-group (related to relationship among different groups) effect on the crowd behaviour. If group has the large number of member, the leader-follower structure runs more efficiently and smoothly than clustered structure [6].

In [4], authors observe that group behaviours (stay with or searching for other group members) have influences on the choosing of exit doors. People in group choose the regular exit (front entrance, bar exit) more than individual people that have used for instance the available windows to exit. So, they conclude that agents belonging to a group run away together. [7] demonstrates the significance of knowledge, social behaviors and incorporating perception of occupant in the simulation of disaster.

On the social side, a lot of works are conducted that can help to more understand the behavior of people in emergency situations. Social interactions are extremely common and strongly influence the response seen in real evacuations [8]. In an emergency situation, people use their past experiences in crisis such as knowledge (the familiarity with the place) and their perceptions to decide what to do [9]. They try to choose the familiar way to escape instead of discovery new ones.

A lot of reports (see [10] for instance) show that when the danger increases, the mutual aid between persons exposed to this danger also increases, they share emotions and information, etc. [11], [12]. Note that more help comes from man than woman [10], [13], [14] but women are more likely to alert others [14]. There are only a few cases of selfish behaviors. One of the faces of this mutual aid is the constitution of groups of persons that try to stay together each time it is possible [15]. Separation from family members causes the stressful event more than threat of injury [9]. Due to these reasons, the families often delay emergency evacuation until all members are close and safety [16]. People in emergency situations try to have the same actions as others (coping mechanism) rather than being passive victims [9], [17]. Sociological studies show that groups increase chances to be saved [17].

\section{B. Emotion, Group, Decision-making}

Emotion has been studied for a long time in psychology, philosophy and more recently in cognitive sciences [18]-[21]. All these works stress the importance of emotion in decisionmaking process, especially when we need to react in a very short time (that is the case in crisis situation). So, recent works show that emotion is very important in the understanding of crisis situations [10], [17], [22], [23] such as fire, disaster, etc. In [24], Damasio shows that a lack of emotion may entail a poor decision making process and an irrational behavior.

In the simulation area, lot of works focus more specifically on emotion spreading [23], [25]-[27]. In [27] for instance, the authors present simulations about relationships between emotion, information and belief. All members of a group can be influenced by emotions of other members of this group.
There is a lot of review of the evacuation simulation, emotion and decision making in recent decades [6], [28]-[32], but relations between emotion and decision making process remains unclear. In [16], ESCAPES (that is an evacuation simulation) is introduced. This simulation takes into account emotion and social groups such as family. Many actions of families are modeled and simulated such as follow or find parents. Note the simulation is about Tom Bradley International Terminal (TBIT) at Los Angeles International Airport. The higher fear level of emotion causes the higher speed of moving, so causes the higher collision. The emotion diffuses among the people and also the authorities and securities who have the low fear level (calm) and takes the leadership role. The fear level of agent increases when neighbours have the higher fear, and decrease when agent pass by authority or security. We see that this is a simple mechanism of emotion contagion and the authors don't explain how emotion spread and how calculate the fear level of emotion? We think that the changing of emotion in crisis is more complicate than that. The authors give the only simple influence of emotion on the action of agent as the speed, and there is no link of emotion with the group evacuation and decision making of agent.

Based on these analyses, we summary in the Table I related works that study the effect of group and emotion in decision making during emergency evacuation. Regarding these related works, in terms of group behavior, we propose a formalization of all identified social interactions in emergency evacuation as presented in Section 3. We also advance further in the modeling of the influence of social interactions and emotion on decision-making of occupants in emergency evacuation as presented in Section 4.

TABLE I. SUMMARY OF RELATED WORKS REGARDING THE EFFECT OF GROUP AND EMOTION IN DECISION MAKING DURING EVACUATION

\begin{tabular}{|l|c|c|}
\hline Models & group & emotion \\
\hline Bosse et al. [25] & & $\checkmark$ \\
Durupinar et al. [26], [33] & & $\checkmark$ \\
Fahy [34] & & \\
Ketchell et al. [35] & $\checkmark$ & \\
Kisko et al. [36] & & \\
Korhonen et al. [37] & $\checkmark$ & \\
Kuligowski et al. [28] & & $\checkmark$ \\
Le et al. [38] & $\checkmark$ & \\
Ling et al. [4], [5], [7] & $\checkmark$ & \\
Murakami et al. [39] & \\
Nguyen et al. [1], [40], [41] & & \\
Nguyen et al. [42] & $\checkmark$ & $\checkmark$ \\
Qiu and Hu [3] & $\checkmark$ & \\
Santos and Aguirre [6] & $\checkmark$ & \\
Ta et al. [2] & & $\checkmark$ \\
Thompson et al. [43], [44] & & \\
Tsai et al. [16] & $\checkmark$ & $\checkmark$ \\
\hline Our model & $\checkmark$ & $\checkmark$ \\
\hline
\end{tabular}

\section{FORMALIZATION OF GROUP BEHAVIORS IN EMERGENCY SITUATION}

\section{A. Types of Group}

1) Social group: We define a social group as a gathering of persons that have pre-existing social relationships, such as members of a family, a group of friends, a group of colleagues. In emergency situations, members of social group discuss, help each others and move to the exit together. People can place themselves in dangerous situations to search others even in a developing threat [15]. 
Social groups are defined at the beginning of the simulation and remain unchanged. In emergency situations, social group members may not to be at the same physical place at a given time. (For instance, a group member is at the rest room.) In this case, the social group still exists.

To formalize all social groups we let a list socialGroup. To distinct social groups, we define a id variable of social group of agent $_{j}$ is $i_{\text {ssocialGroup }}$ agent $_{j}$. We identify a social group:

$$
\begin{array}{r}
\text { socialGroup }_{i}=\left\{\text { agent }_{j} \in C U S T O M E R:\right. \\
\\
\text { idsocialGroup } \left._{\text {agent }_{j}}=i\right\}
\end{array}
$$

We identify two concepts of group that are the social physical groups and the physical groups. While a social group represents the relationship among individuals, these two groups represent the physical distance between them.

2) Social physical group: A social physical group is a group of persons who have social relationship and navigate together. With this definition, a whole or a part of a social group can be considered as a social physical group during the crisis if there are at least two members navigating together. A social group can be composed of one or many social physical groups. We call a leader of a social physical group a social leader. The followers of a social physical group are the social followers. The social followers always follow their social leader.

We formalize this concept as:

$$
\begin{aligned}
& \text { socialPhysicalGroup }_{i}^{t}= \\
& \left\{\text { SOCIALFOLLOWERS }_{i}^{t} \cup \text { socialLeader }_{i}:\right. \\
& \forall \text { socialFollower }_{j} \in \text { SOCI ALFOLLOWERS }_{i}^{t}: \\
& \text { idsocialGroup } \left._{\text {socialFollower }_{j}}=\text { idsocialGroup }_{\text {socialLeader }_{i}}\right\}
\end{aligned}
$$

All followers in SOCIALFOLLOWERS $S_{i}^{t}$ at the time $t$ are leaded by a socialLeader . $_{\text {. }}$

3) Physical group: We define a physical group is a group of persons who navigate side by side, leaded by a leader and all members have no social relationship with others. A leader of this group could be a customer or a security agent. Other members of this physical group are followers of this leader. With this definition, a physical group cannot contain more than one member of social group. A social leader or an isolated person could be a follower or a leader of a physical group. But the social followers are not a part of a physical group because they are always lead by a social leader.

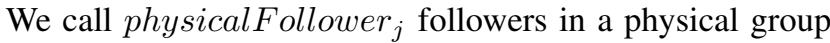
and physicalLeader $_{i}$ a leader of this physical group. We formalize this group as:

$$
\begin{aligned}
& \text { physicalGroup }_{i}^{t}= \\
& \text { FOLLOWERS }_{i} \cup \text { physicalLeader }_{i}: \\
& \text { pphysicalFollower }_{j}, \\
& \forall \text { physicalFollower }_{k} \in \text { SOCIALFOLLOWERS }_{i}^{t}, \\
& \text { idsocialGroup }_{\text {physicalFollower }_{j}} \\
& \neq \text { idsocialGroup }_{\text {physicalLeader }_{i}} \text { and } \\
& \text { idsocialGroup }_{\text {physicalFollower }_{j}} \\
& \left.\neq \text { idsocialGroup }_{\text {physicalFollower }_{k}}\right\}
\end{aligned}
$$

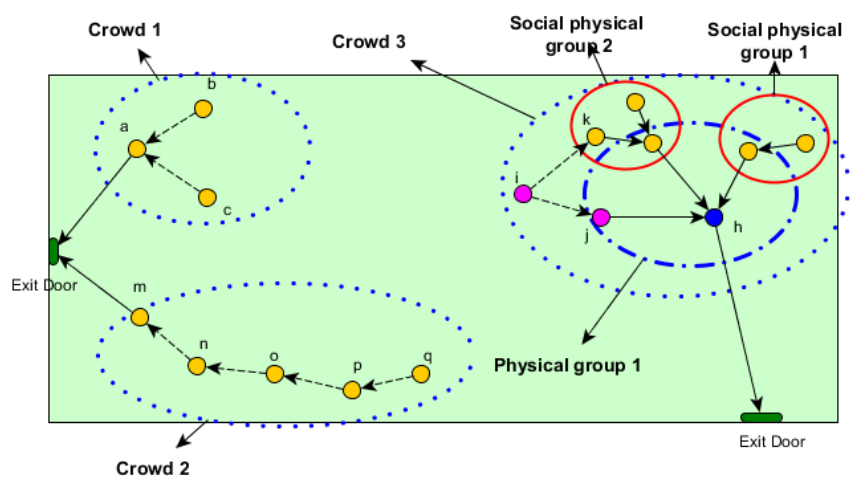

Fig. 1. Some cases of crowd in our model.

By definition, $i \in$ physicalGroupt $i_{i}^{t}$ and then, physicalGroupt $i_{i} \neq \emptyset$.

4) Crowd: In emergencies, people tend to characterize themselves as a member of one or many groups. Or they just follow a group of people and are not leaded by anyone else. Many researchers argue that there exist the herding behaviour during the crisis. It is difficult to give an exact definition of crowd. We define crowd is a group of persons who navigate with the same way and it exists one person in that group who has a physical relation with at least two persons and one stranger. We identify agent $t_{i}$ that has a physical relation with agent $_{j}$ if and only if agent $t_{i}$ leads agent $_{j}$ or agent $_{i}$ follows agent $_{j}$, or agent ${ }_{j}$ moves based on the direction or the position of agent $_{i}$. Any person who has any physical relation with any member in the crowd belongs to this crowd. With this definition, a crowd has at least three persons and one stranger, and a social physical group or a physical group is not a crowd. But a social physical group navigating with one individual could create a crowd. Or a physical group navigating with an individual who does not follow a leader but moves based on a follower position could create a crowd. Or a crowd could be created by many social physical groups with many physical groups when existing one physical relation among them.

Some cases of crowd are described in Fig. 1. In crowd $d_{1}^{t}$, agent $_{b}$ and agent $_{c}$ navigate based on agent $_{a}$ but agent $_{a}$ is not a leader of both of them. In crowd $_{2}^{t}$, each agent navigates based on another one such as agent ${ }_{n}$ navigates based on agent $_{m}$, and agent $_{o}$ navigates based on agent ${ }_{n}$ etc. crowd $_{3}^{t}$ is a little complicated. There are two social groups with their leaders following a physical leader agent $_{h}$. Individual agent $_{j}$ follows this leader too. While another individual agent $_{i}$ navigates based on a follower of a social group agent $_{k}$ and individual agent $_{j}$. A crowd could be created by the social group socialGroup $_{1}$ and the physical leader physicalLeader agent $_{h}$, or by the social group socialGroup $_{1}$ and individual agent ${ }_{i}$, but all other agents have a physical relation with at least one agent, so crowd $_{3}^{t}$ is represented by a group of agents like in Fig. 1 .

We suppose it exists three agents: agent $_{d}$, agent $_{e}$, agent $_{f}$ including in Fig. 1 (see Fig. 2). agent $_{f}$ can observe three agents agent ${ }_{d}$ agent $_{e}$, agent $_{i}$, but they do not follow the same way, agent $t_{i}$ navigates to the door $_{2}$ while agent ${ }_{d}$, agent $_{e}$ 


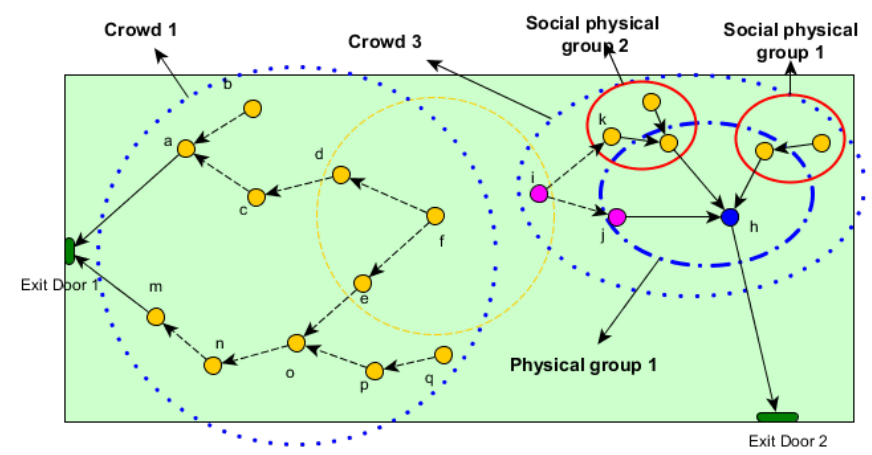

Fig. 2. Herding behaviour in crowd.

navigate to the door $_{1}$. Now agent $f$ triggers herding behaviour and navigates based on agent , $_{\text {agent }}$ (note that both of them are not leaders of agent $_{f}$ ). With the definition of crowd, two crowds crowd ${ }_{1}^{t}$ and crowd $d_{2}^{t}$ are merged to one only if it exists a physical relation among them. If it exists only two agents in perception region of agent ${ }_{f}\left(\right.$ agent $_{d}$ and agent $\left._{i}\right)$, agent $t_{f}$ will choose a nearest agent to follow or choose randomly in case the distances to them are equal.

We formalize crowd $i$ at time $t$ :

$$
\begin{aligned}
& \operatorname{crowd}_{i}^{t}=\left\{C R O W D_{i}^{t}:\right. \\
& \exists\left(\text { agent }_{a}, \text { agent }_{b}, \text { agent }_{c}\right) \in C R O W D_{i}^{t}, \\
& i_{\text {idsocialGroup }} \text { agent }_{a} \neq i_{\text {socialGroup }} \text { agent }_{b} \\
& \text { or } i_{\text {dsocialGroup }} \text { agent }_{a} \neq i d \text { socialGroup }_{\text {agent }} \\
& \text { and } \exists \text { Relation }\left(\text { agent }_{a} \text { agent }_{b}\right)^{t} \\
& \text { and } \exists \text { Relation }\left(\text { agent }_{a} \text { agent }_{b}\right)^{t} \text {, } \\
& \text { and } \text { tphysicalLeader }_{\text {agent }_{b}} \neq \text { physicalLeader }_{\text {agent }} \\
& \text { agent }_{d} \in C R O W D_{i}^{t}, \exists \text { agent }_{e}: \\
& \left.\exists \text { Relation }\left(\text { agent }_{d} \text { agent }_{e}\right)^{t}\right\}
\end{aligned}
$$

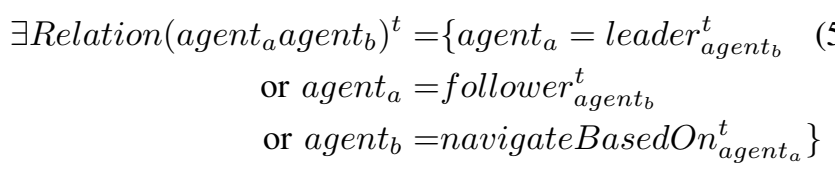

In a crowd, there may exist or not a leader. But there are always people who drive this crowd to navigate to exit door.

\section{B. Group Attributes}

1) Identification: The identification helps control the social and physical relations in evacuation. To control social groups, as we describe above, we need a social identification $i_{\text {idsocialGroup }}$ for social group $i$. All members in a social

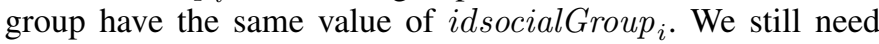
another identification to manage a physical movement in a physical group or a social physical group. So, the other

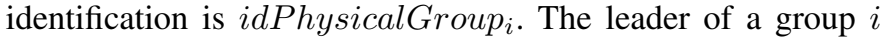
(social leader and physical leader) and his/her followers have

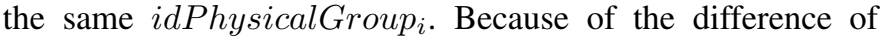
identification of each agent, we can set idPhysicalGroup $i$ $=i d$ Agent $t_{\text {leader }}$.
2) Types of social group: Each social group has a different relationship, such as: a group of families has the strongest relationship among the members, while the relationship of members in group of friends is weaker, and similarly with relationship in group of colleague.

In our model, we formalize the type of a social group $i$ :

typesocialGroup $_{i}=\{$ family, friend, colleague $\}$.

3) Moving speed: We define walking speeds for individual agents. Generally, the walking speed of each agent of a group is different. We consider here the "group effect" that causes some persons will walk faster than their normal walking speed and some others will walk slower than their normal walking speed. An explanation is that the slowest persons will be helped and motivated by others. Conversely, the fastest persons will spend time to help the slowest persons. We use term Group for both PhysicalGroup and SocialPhysicalGroup. So, we define the group walking speed speed group $_{i}^{t}$ of a group $t_{i}^{t}$ at time $t$ as an average speed:

$$
\text { speed }_{\text {group }_{i}^{t}}^{t}=\frac{\sum_{j \in \text { group }_{i}^{t}} \text { speed }_{j}^{t}}{\text { card }_{\text {group }}^{t}}
$$

where, $\operatorname{card}_{\text {group }}^{t}$ is the number of agents in $\operatorname{group}_{i}^{t}$.

Now, we could suppose that the current walking speed of agents that are member of a group is the group walking speed. But what happens if we suppose that this speed is greater than the maximal speed of some agents? We adopt the following heuristics. For every $i \in L E A D E R$ :

$$
\text { speed }_{i}^{t}= \begin{cases}\text { speed }_{i}^{\text {norm }} & \text { if leader } \\ \min \left(\text { speed }_{i}^{\text {max }}, \text { speed }_{\text {group }_{i}^{t}}^{t}\right) & \text { else }\end{cases}
$$

and for every $i \in C U S T O M E R$ :

speed $_{i}^{t}= \begin{cases}\operatorname{speed}_{i}^{\text {norm }} & \text { if leader } \\ \min _{i}^{t}=\emptyset \\ \left.\text { speed }_{i}^{\text {max }}, \text { speed }_{\text {group }_{\text {leader }}^{t}}^{t}\right) & \text { else }\end{cases}$

On one hand, according to this definition, some agents may walk slower than other members of the group and then, these slowest agents can lose the group (they are removed from this group). On the other hand, the faster an agent walks, the more it will probably be a survival. An important consequence of that is to find a "good" group walking speed, that is, the speed that will allow saving most of the humans as possible. This is an important question. We have tested a lot of group walking speeds by using scale. speed $_{\text {group }_{i}^{t}}^{t}$ instead of speed $_{\text {group }}^{t}$ for scale $_{s} \in[0.0,2.0]$.

4) Leader: Each group $t$ has only one leader at time $t$. Everyone else in this group are the followers of him. The leader can do some actions such as: maintaining group, waiting members, exchanging information, supporting moving. A leader by definition belongs to a physical group or a social physical group. There is no definition of leader for social group, because the leader is created in the evacuation process when members navigate together. We identify a leader of a social physical group is socialLeader $i$ and leader of physical group is physicalLeader . $_{\text {. }}$. 
5) Followers: Followers in a group are all members in this group except the leader. Followers follow the leader and can receive support from him. Note that there exists agents navigating based on other agents, but they are not follower, because there is no leader among these agents.

\section{Individual Attributes Related to Groups}

1) Roles in physical and social physical groups: With no influence of emotion, we suppose that in a fixed group with no other person joining to, the role of a person is fixed. That means, a leader can not exchange his/her role for a follower. The process of exchanging the role may occurs when the group has a new member. Now we will present in detail the roles of agent in group and the transition between them.

The roles of people who has a social relationship:

- Isolated belongs to a social group, but navigates alone (like an individual). Not likely as individual persons, isolated person may search other members before decide to exit or he/she will join with his/her members in the social physical group when he/she perceives them. Isolated person is an individual when he/she is not in the searching members process and does not see other social members. In this case, he/she can follow other strangers to become a follower or lead other people in a physical group. When he/she joins to a social physical group, he/she may become a social leader or a social follower.

- Social leader is a leader of a social physical group. As we know in reality, the leader is a person who has more knowledge, more experience and has particular characters that influence others more than anyone else in this group. So, we suppose that a leader is a person who has the highest value of groupInfluence in this group.

Similarly to isolated person, a social leader can take a role of a physical leader or physical follower in a physical group when he/she joins to this group, or just navigates as an individual agent. When another missing social member join to a social physical group, a social leader may change the role to a social follower depending on the value of groupInfluence of the new member. When navigating in a physical group, he/she always follows social members having higher priority than that of strangers. Example, he/she can leave from a physical group to maintain his/her social physical group or can help his/her social followers before helping strangers.

- Social follower follows his/her social leader. He/she always follows his/her social leader who has a highest priority. Although his/her leader can follow another people. Social follower can become an isolated people when he/she misses his/her social leader (caused by obstacle or neighbours). As described above, isolated people and social leader can participate in a physical group, but not for social followers.

The role of people in physical group:

- Physical leader leads all his/her physical followers. We based on the value of groupInfluence in (9) to decide which person is a leader. A physical leader can become a follower if an individual having the higher groupInfluence joins to the group, or an individual when he/she misses all his/her followers. A physical leader navigates as an individual to find an exit door or navigates following other agents. A physical leader acts to maintain or helps members in the group, but the relation among them is not lower than that of the members in a social physical group. Example, his/her waiting time could be less and the maintenance distance could be more than these values of social leader.

- Physical follower is leaded by a physical leader. Each physical follower has only one physical leader and can receive support from him or from other members in the group.

2) Group influence: This attribute influences directly the role of people in the group. That may be a complicated attribute because it depends on many factors. But we think there are three main factors influencing its value: their characters (age, strength, sex, empathetic, etc.), their knowledge about exit doors and their experiences in the crisis, the relationship with all others members in the group. We formalize this value as in 9:

$$
\begin{aligned}
\text { groupInfluence }= & \frac{1}{3}\left(\begin{array}{l}
\text { Leader } \\
\text { character }
\end{array}+\right. \\
& \text { fLeader } \\
\text { knowledge } & \left.+f \text { Leader }_{\text {member }}\right)
\end{aligned}
$$

All values of groupInfluence, fLeaderCharacter, $f$ Leader Knowledge, fLeaderMember are in $[0,1]$.

3) Waiting time: When missing a member of a group, a leader tries to wait his/her follower before continuing navigate to the target. When the physical leader stands, all other members wait too because they follow their leader. This duration depends on the emotion intensity and also the relationship among the members of group. The social leader has a higher value of waiting time than that of the physical leader, and it is similarly to the leader of group of families and group of friends.

We use a variable waitingTime to represent this attribute. waitingTime $=f($ emotion, , relationship $)$

4) Minimal distance to maintenance of group: This variable is needed for the group maintaining action. The leader can wait or go back to the followers to aid. We suppose that the value of this variable depends on the emotion and also the relationship among leader and followers, maintainDistance $=f$ (emotion, relationship $)$. The go back action will be triggered when the distance between a member and his/her leader is higher than gobackDistance and the waiting action will be triggered in case that this distance is higher than waitingDistance but lower than gobackDistance.

5) Searching time: In emergencies, people try to search other members in a social group before exiting. And depending on the type of social group, the searching duration may be different. The families usually take long time to search other members than group of friends. We suppose this occurred when the emergency starts. The isolated person and social leader take a searching time searchingTime to search each 


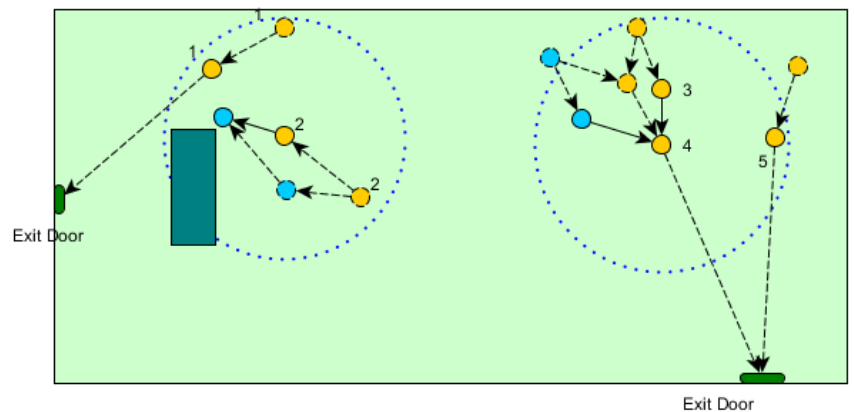

Fig. 3. Agents in social group regroup when they see each other.

others. Similarly to waitingTime, this duration depends on the emotion and relationship between them searchingTime $=f($ emotion, relationship $)$. The higher fear intensity causes the lower searching time and lower waiting time.

\section{Action of Agents in Group}

1) Regrouping: We suppose that some isolated persons cannot find other members of a social group in a searchingTime. So, they navigate to the exit door. But, in the evacuation process, they see each other. In this case, they will trigger the regrouping action to create or update members of this social physical group. Example in Fig. 3,

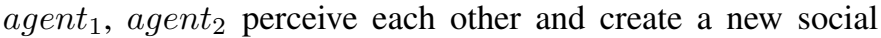
physical group. Another case, agent ${ }_{3}$, agent $_{4}$ creates a social physical group, but while moving to the exit door, a social leader socialLeader $_{4}$ and agent $_{5}$ see each other. We suppose that groupInfluence $_{\text {agent }_{5}} \&$ groupInfluence $_{\text {agent }_{4}}$, so the agent $_{5}$ is a new leader of this social physical group and he/she is also the leader of this physical group (the pink agent is a stranger regarding to three agents: agent $_{3}$, agent 4 , agent a $_{5}$. Now, the followers of agent 4 will follow agent . $_{4}$

As we denoted above, social followers always follow a social leader, so we do not need to care about them in the regrouping process because they do not change their role. Only isolated person and social leader can change their role. So, if they find the members, they will compare the value of groupInfluence. The agent with higher groupInfluence will become a leader of this group. If the old leader becomes a social follower, he/she assigns all his/her followers to the new one. Now the new leader has the list of all social followers of this group.

2) Searching other members in group: This action is taken by the people who are in a social group. That means when the emergency begins, all members in a social group will try to find other members during a searchingTime. They can explore the space to find members, or reach to the meeting point and the last position where they met. It depends on the strategies of people. In case that there are all members or the searching time is over, they (social leaders or isolated persons) will navigate to exit. Fig. 4 represents the searching member action based on the meeting point.

agent $_{1}$, agent 2, agent $_{3}$ reach to the meeting position to try to search other missing members. The agent $_{4}$ is a social

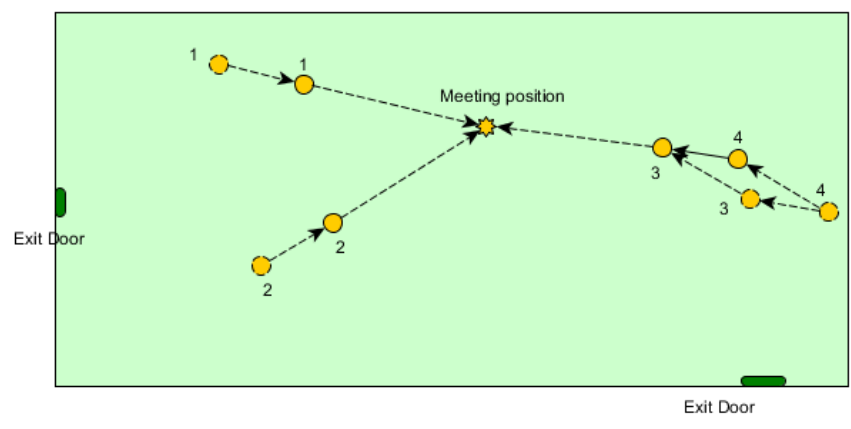

Fig. 4. Searching members before exiting.

follower, just follows the social leader agent $_{3}$, while agent $t_{1}$, agent $_{2}$ are isolated agents.

As we presented above, the searchingTime can be influenced by the emotion and relation among members in a group. In a group of families, this duration will be higher than in the group of friends. If an agent has a high fearLevel the duration of searching will be reduced.

We suppose that in searching other social members, people do not participate in a physical group or do the action linked with a physical group such as: merging groups or maintaining group. But they can still help exchanging information to other persons.

Similarly to the regrouping action, we check only with the isolated persons and social leaders for this action. We need to verity the simulation time that must be lower than the searching time. In the searching time, agent explores the space, or goes to the meeting point to search missing members. Otherwise, agent navigates by itself to find the way to go out. Agents always check to regroup with other social members during the evacuation process.

3) Merging groups: Merging group is an action only for physical group. That means this action is taken by the stranger people only. An agent navigates as an individual or a physical leader can trigger this action. Note that, a social leader navigates as an individual if he does not join to any physical group. When two agents (leaders of physical group or individual) perceive each other, if they are not independent agents (the agent does not want to follow anyone), they can merge to create a new physical group. Similarly to the social leader, we decide a new physical leader based on the value of groupInfluence. All physical followers (if exist) of two agents will become physical followers of the new leader. This action is represented in Fig. 5.

On the left side, there are two social physical groups with their leaders perceiving each others. We suppose that groupIn fluence $_{1}$ i groupIn fluence g $_{2}$, so agent t $_{2}$ will follow agent $_{1}$. They have both social and physical roles. agent $t_{2}$ is a social leader and physical follower, while agent $t_{1}$ is a social leader and physical leader.

On the right side, agent 4 is a physical leader (before joining to this group, it is an individual) of the group that 


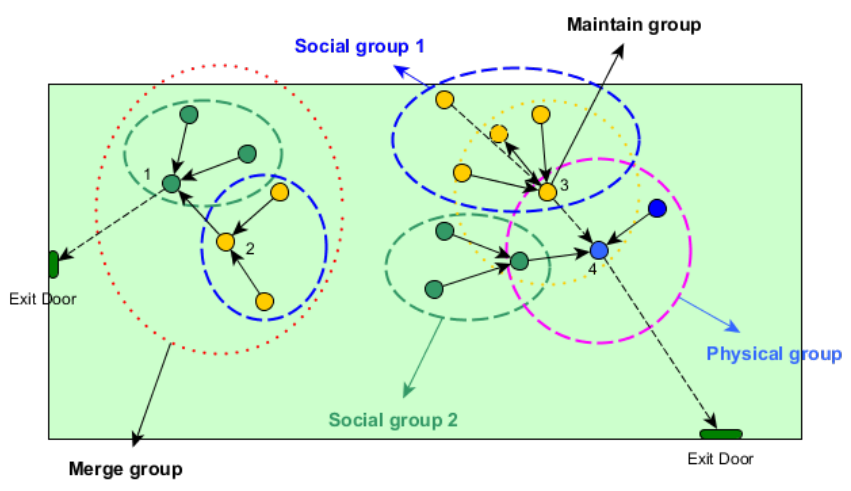

Fig. 5. Merging among group and maintenance group.

has three followers. Two of them are the social leaders, and one is an individual (before joining to this group).

Note that the social leader and the isolated person are assigned automatically the individual role if they navigate by themselves. After that, if they participate in a physical group, their role may change to a physical leader or physical follower. For this algorithm, first we check only for the individual and physical leader because the physical followers do not have influence on the leader role of the group. If they (individual and physical leader) see the new one who has the low independentLevel that means this one wants to join to the physical group. In this case, the groupInfluence will be compared between the old physical leader and the new one to detect the new physical leader. Physical followers will be assigned for the new physical leader (if he/she is different with the old one). The person with high independentLevel can keep navigating alone or become a physical leader, but not a physical follower. The independentLevel is applied only for the physical group but not for the social physical group.

4) Maintaining group: Leaders of social group and of physical group do some actions to maintaining his group such as waiting members of group or going back to support members. Detail of group maintenance is described in Fig. 5. agent $_{3}$ is a social leader of the social physical group 1 , and also a physical follower of physical group. But when missing his social follower, he goes back to search and support this follower. The follower can leave from the physical group if the physical leader does not wait for him.

We need two thresholds to trigger three types of actions in maintaining actions. If the distance between a leader and one follower is higher than the distance of going back and lower than the visual radius, the leader will go back to the center of group of followers. If this distance lower than the distanceGoBack and higher than the distanceOfWaiting, the leader will wait followers until this distance is lower than distanceOfWaiting or the waitingTime is higher than a threshold.

5) Following crowd: The following crowd behaviour is described in [45]: agent can base on the center location of neighbours and average moving direction of them to detect the target. We apply the following crowd behaviour for the agent that has no information of exit door or when it is in

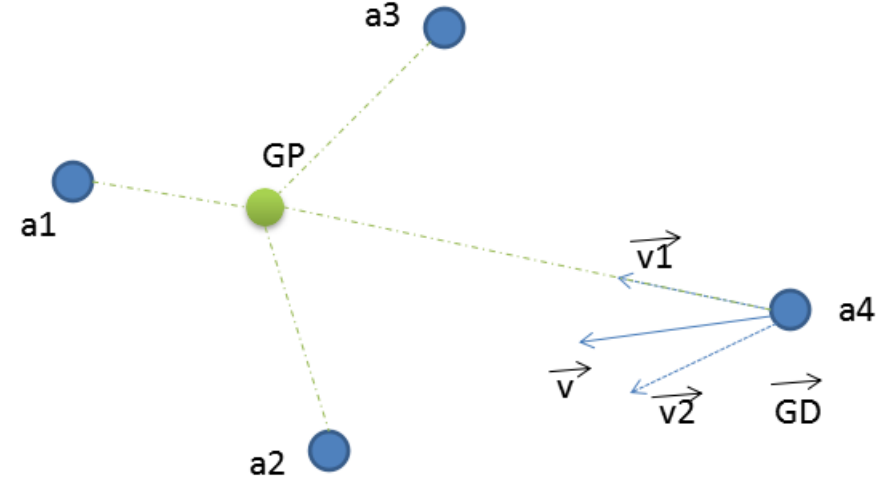

Fig. 6. Leader of group social follow the crowd.

panic. The detail of this action is described in Fig. 6 .

We suppose in the visual region of agent $t_{a 4}$, it exists three agents : agent ${ }_{a 1}$, agent ${ }_{a 2}$, agent ${ }_{a 3}$. The point $G P$ is a center of three agents, and vector vecGD is an average moving vector of these agents. Vector vecunitone and vector vecunittwo are the unit vector of vecagd and vecGD. vecv = vecunitone + vecunittwo is a vector of movement of agent $_{a 4}$.

Following crowd is occurred by individual agents or physical leaders who navigate following some followers. This action is different with merging group, that may occur among individual agents and physical leaders. The priority of merging group will higher than following crowd.

Emotion and knowledge of agent will be checked before triggering this action. The next position will be calculated based on the description above. If this position is not in the moving region, the next position will be automatically generated with the elimination of the position of one follower. This process continues until we find that the next position is on the moving region.

6) Following a leader: A follower follows a leader that means he navigates follow leader's position if they perceive each others. Each follower only follows one leader. It is different with navigating following some persons (or following the crowd that we present below). Example in Fig. 7, in first

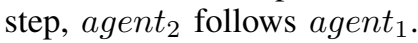

7) Reaching to the last position of leader: When followers loose the leader, they try to reach to the last position of their leader. But, if they cannot perceive the old leader, now they will change the role to the isolated person (if they have the social relationship with another person) or individual person. The example of reaching to the last position of leader is described in Fig. 7. agent $_{2}$ looses his leader agent $_{1}$ because of the obstacle. So, agent ${ }_{2}$ navigates to the last position of agent $_{1}$. At this position, if agent $t_{2}$ cannot perceive agent ${ }_{1}$, he will change his role from a follower to an isolated person depending on his social relationship. If a follower leaves from a group, it may cause the changing role of the leader of this group.

8) Exchanging information among members in group: The process of exchanging information is presented in Fig. 7. The leader can exchange the information with members during the 


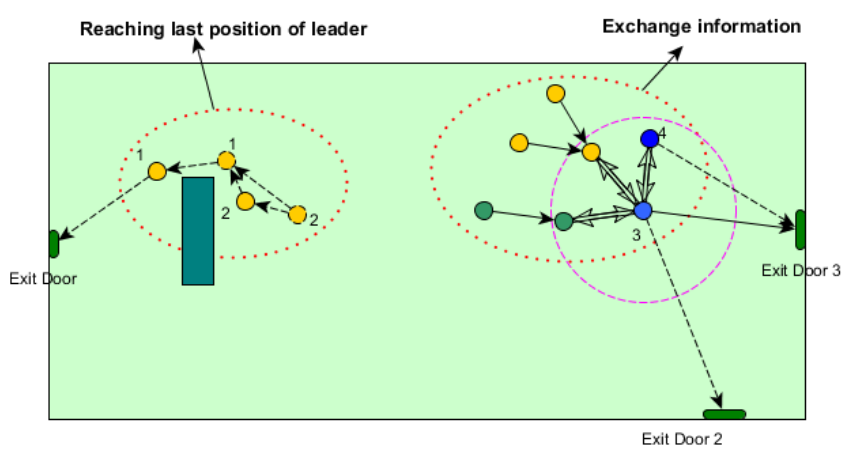

Fig. 7. Reaching to the last position of leader.

evacuation process. Based on this information, the leader may decide to change the old way and reach to the new one that is shorter. The physical leader agent $t_{3}$ first wants to navigate to door ${ }_{2}$. But after exchanging the information with agent 4 who knows the closer $d_{0 o r}$. So, the physical leader agent 3 changes the way and navigates to door ${ }_{3}$. Similarly to the request and diffusion information action of individual agent, this action takes time. That means people have to delay the evacuation while doing this. This action may occur in the social physical and the physical groups. And the leader decides to do this action or not depending on his/her emotion, and the time of exchanging information exchangeTime. Members in group can exchange all known exit doors to each others, or just a few ones depending on their leaving time exchangeTime. The exchangeTime depends on the emergency level. We suppose that it takes a unit time unitTimeExchange to exchange one exit door for all members in the group.

To trigger this action, the knowledge of all followers must be different with the one of the leader. So, first we have to detect the known exit door list of all followers, and then check if a leader is available (has time) to exchange the information or not. If he (she) has time, all members will wait and exchange the information. The known exit door will be diffused for all members in the group. This action may occur many times with many groups, so we set total time for exchanging information for each agent in the initiation of simulation. And if agent uses over this duration, it will not participate in this action.

9) Following the guidance of security agent: Security agents in emergencies take an important role. They are the people who know all about the place such as exit doors, and also the information about the congestion of evacuation, etc. Moreover, the security agent can force customers respect the social norms. These persons can guide the shortest way or help (moving support) people to exit. We suppose all customers with the role related to physical groups (such as physical leader, physical follower, individual), have to follow security agents or follow their guidance. Note that, social followers always follow their social leader. A security agent takes the role of the physical leader in evacuation but not the social leader. We suppose that these security agents will help customer in a helping time helpingTime. This value depends on the crisis level. And we suppose that all security agents have the same and highest groupInfluence.

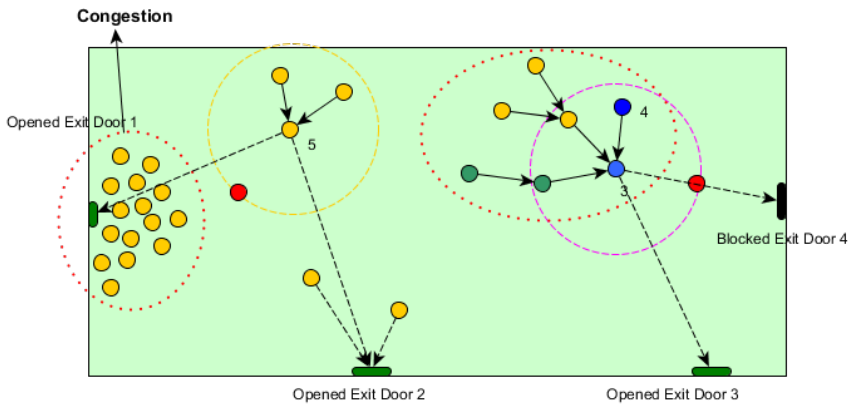

Fig. 8. Following the guidance of security agent.

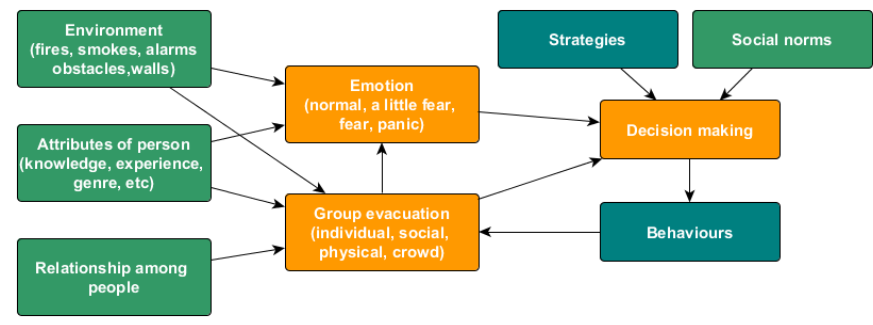

Fig. 9. Relation among emotion, group evacuation and decision making.

Fig. 8 presents the guidance of security agents (with red color) in case of congestion and blocking the exit door. The physical leader agent $_{3}$ changes his direction when receiving the guidance from security agents. Or group of agent $_{5}$ changes the direction to navigate to the other door 2 caused by the congestion near the door 1 .

When seeing the security agents, customers will know if a security agent is in the guiding process or in the evacuation process. If the security agent is in guiding process, customers will follow his/her guidance to exit. If a security agent is in the evacuation process, now the physical leader role will be assigned automatically to him/her, and all individuals, physical leaders, physical followers have to follow him.

\section{EMOTION AND DECISION MAKING}

\section{A. Introduction}

In the previous section, we present the attributes, the actions of the individual agent and the agents in group, also their emotion during emergencies. As we known, agents have their own actions that depend on the existence of relationship with other agents or not. Emotion also has influence on the actions of agents. But which one will drive the actions of agent? We suppose there exist a phase based on the group evacuation and the emotion, that is decision making. This phase may based on the strategies and other conditions such as social norms to decide the behaviour of agents. The detail is represented in Fig. 9.

Strategies component is understood as an artificial intelligent module of agent. It provides methods for agent to leave from the crisis. When an agent does not know about the exit door, it can follow one direction and then follow the wall to find an exit door. Or when the exit door is blocked, 
agent can automatically follow the wall or navigate directly to another exit doors. We provide agent a simple strategy to avoiding obstacles. We can make the agent more intelligent by extend this module. With different environments such as in the supermarket, in the airport, in the office building, the plan are different, so we need different strategies for agent to evacuate from crisis. Example with the case of supermarket, agents can observe the environment, they can choose following the wall or following the border of obstacle to exit. With the office building, we cannot apply this strategies because of different plan. Now, the obstacle can be the wall of the office, so applying actions such as following the wall or following one direction are not effective. Or with the airport, the space of moving is narrow but long and there are not many obstacles on the way of moving. And the exit door numbers are not the same in the super market, we may passing many exit door to quit the airport. In this case, we may apply the following exit sign to navigate strategy.

The green components such as: Environment, Attributes of person, Relationship among people, Social norms are independent with Emotion and Group evacuation modules (except knowledge of exit door, it can be updated by exchanging information among others during evacuation process). That means, these components are not influenced by the emotion and the evacuation process. The emotion depends on the environment, attributes of agents and the group evacuation (neighbours).

Social norms provides the rules that people should follow such as following the queue to exit, moving to help handicaps, children, elder, etc. during the emergency evacuation.

With different fear levels, actions of agent may be different. But, caused by the various of fear levels in $[0,1]$, we need to divide by the state of emotion to easily control behaviours and actions of agent. There are four states of emotion of agent are: stateNormal, stateAlittleFear, stateFear, statePanic. The relations between the emotion states and the behaviours of agent are presented in Fig. 10. Example, when people is in panic, they only reach to the red behaviours and actions and do not do any other. But, depending on the other components such as Strategies and relationship with others in evacuation process (see social members, see security agent, etc.), Social norms, Decision making module will decide the final behaviour of agent. Similarly to stateAlittleFear, stateFear states, they influence the time of blue actions such as the waiting time, the searching time, the exchanging information time, etc.

There are four types of behaviour of agent in the evacuation. In this section, we will define more clearly these behaviours and actions of agent for each behaviour. The priority behaviours of agents in group evacuation is presented in Fig. 11.

If they are the agents in the social group, they take the social group behaviours with the highest priority. And then they adopt the physical group behaviour or crowd behaviour to navigate to the exit. In the case they do not see others or they have the high independent level, they can navigate by themselves and adopt individual behaviour. Note that, the leaders of social physical group and leader of physical group do the actions to maintaining their group but they navigate

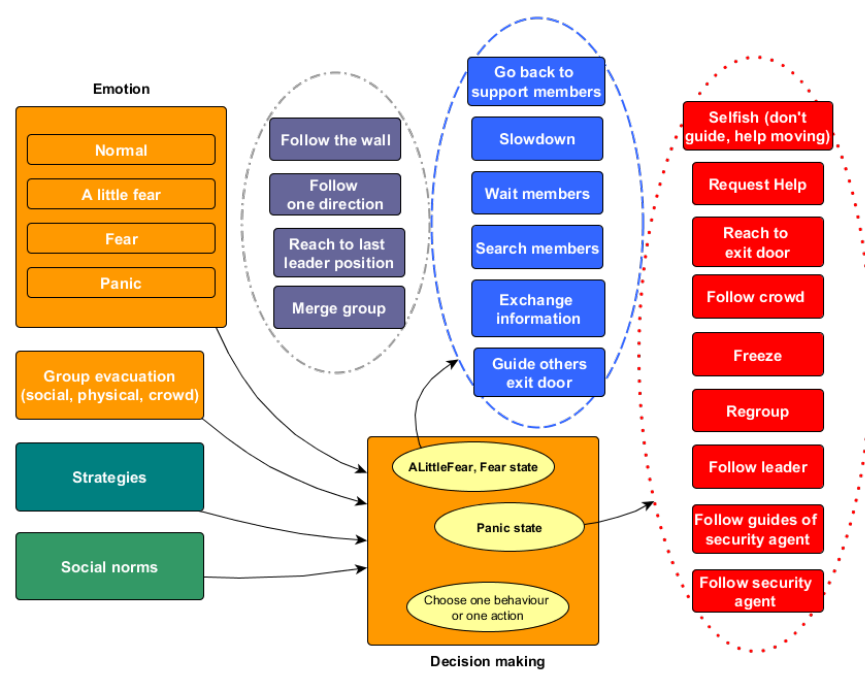

Fig. 10. Relation between the emotion states, behaviours, actions of agent.

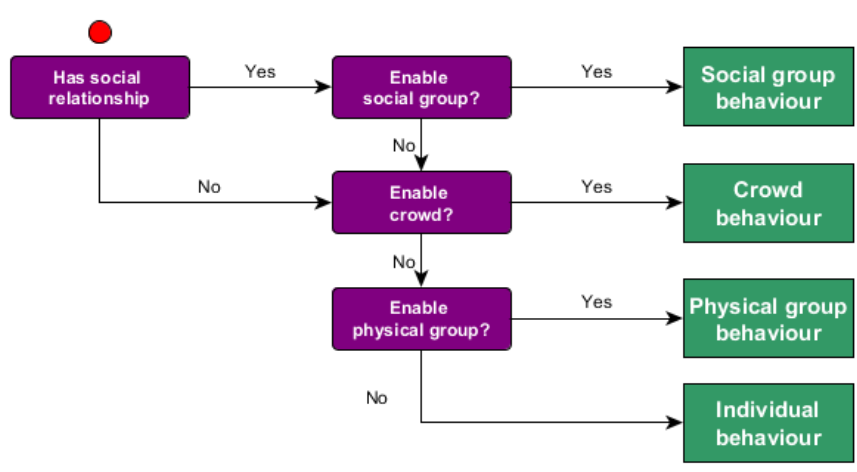

Fig. 11. Priority behaviours of agents.

by themselves to the exit (they do not follow others). So they adopt the individual behaviour. We will present in detail actions of agent in social physical group, in physical group and of individual agents in the following section.

\section{B. Decision Making of Agent in Social Group}

There exists three roles of agent in social group: isolated, social leader and social follower. While the isolated agents move alone, the social leaders and social followers move in the group. If isolated agents join to a social physical group, their role will be changed to social leader or social follower.

1) Isolated: The action decision-tree of isolated agent is presented in Fig. 12.

In each step, isolated agents will detect if they can see new isolated agents or social leaders who are in the same social group. If they do not see any other members in the social group, they will calculate the searching time existing. The searching time is limited and different with each social group. For all agents in the same social group, they have the same value of searching time. All agents in a social groups will try to reach to other members in this duration. If they do not find anyone else, they will start to navigate by themselves 


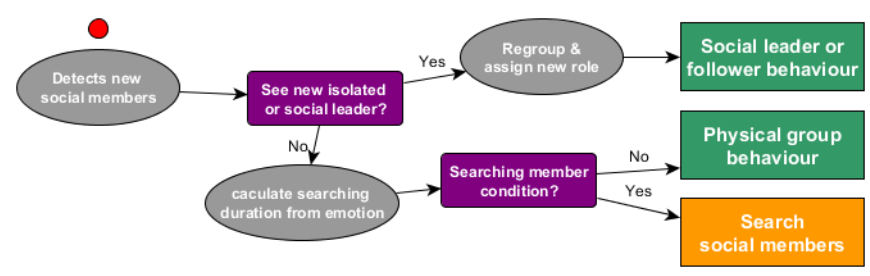

Fig. 12. Action decision-tree of isolated agent.

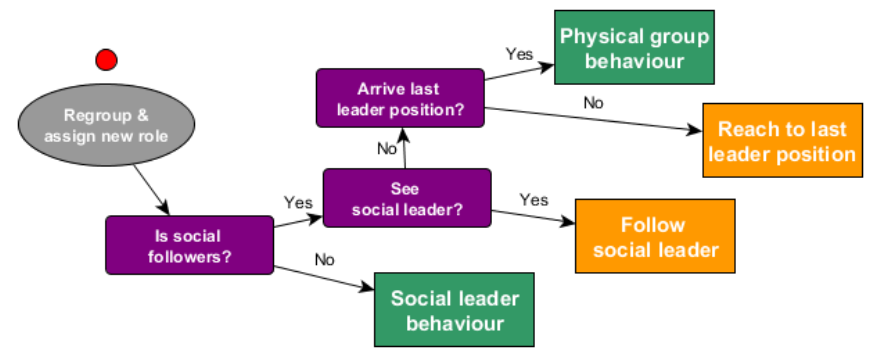

Fig. 13. Action decision-tree of social follower agent.

to the exit door. If isolated agents see other members, they will regroup with them to create a new social physical group. In this case, the social role of agent change. If isolated agents do not see and regroup with social members and are not in the searching members process, they navigate as an individual and can do the actions related to the physical group.

2) Social follower: The action decision-tree of social follower is represented in Fig. 13.

In a social physical group, there exist only one social leader and one or many social follower(s). And the social followers always follow the social leader if they see their leader. Caused by the different speeds of agent and avoiding obstacle process, the social followers sometimes lose their social leader. In this case, they will try to reach to the last position of their social leader. If they do not see their social leader when arriving this position, they will decide to navigate as isolated agents as we describe above. That means, they change their role from the social follower to the isolated one.

3) Social leader: The action decision-tree of social leader is presented in Fig. 14.

The action of social leaders are more complicated than

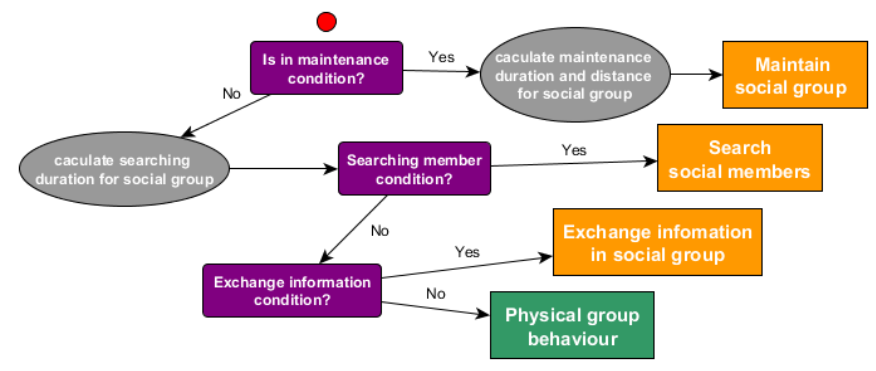

Fig. 14. Action decision-tree of social leader agent.

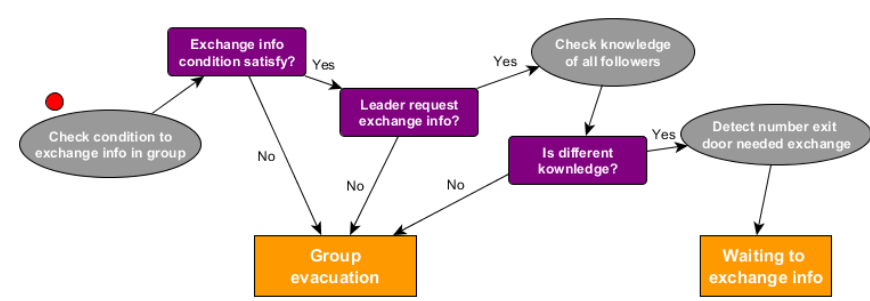

Fig. 15. Exchange information decision-tree in group.

the ones of the social followers. They act as isolated agent (searching members, regrouping with other new members, navigating by themselves) and also do the maintaining actions and exchanging information among the members in group. During the evacuation process, if the distance between the social leader and his/her followers is higher than some thresholds or some social followers are missing from group, the social leader will trigger the maintaining action. He/she can slow down, go back to the social followers position or wait the missing members in a waiting time. If the social leaders are not in the maintaining conditions, they will verify if they are in the searching member conditions or not. If yes, they will lead all their followers to search other social members. If they have no time to searching, they continue to check if they have time to discuss with all followers to exchange the information or not. If they have time, all members in group will stop moving to exchange the information, and then, they find the best way to exit based on the knowledge of the social leader.

We suppose that the social leaders control the exchanging information process. That means, they decide to do this action or not. And we suppose that the exchanging information process takes time, so all members in the group have to stay in a location and do not do other actions. During this process, all members will receive the same information. Example, agent ${ }_{i}$ knows one exit door $\left(\right.$ door $\left._{1}\right)$ that no one knows, and after the exchanging information process with the social leader, all members in group know the position of door 1 .

If social leaders do not do the action related to the social group, they will navigate as individual agents and do the actions of the physical group (similar with isolated agent).

The detail of exchanging information in group is presented by the decision-tree in Fig. 15.

Firstly, a leader will check if he/she is satisfied with the conditions of exchanging information action in the group (such as, he/she does not do another action as searching members or maintaining group). If not satisfied, he/she will continue to do these actions. If yes, the leader will continue verify if he/she can request the exchange information action with all members in group (he/she has to have the exchanging time and is not in another exchanging information process). If no, the group continue following its action. If yes, we suppose that the difference between the exit door lists of followers and of the leader will be recalculated. If there is no difference, all members in the group continue doing their actions. Otherwise, they will wait until the information exchanging finished. The information exchanging causes the delay of evacuation, but it gives the chance to reach to the nearest exit door if the leader 


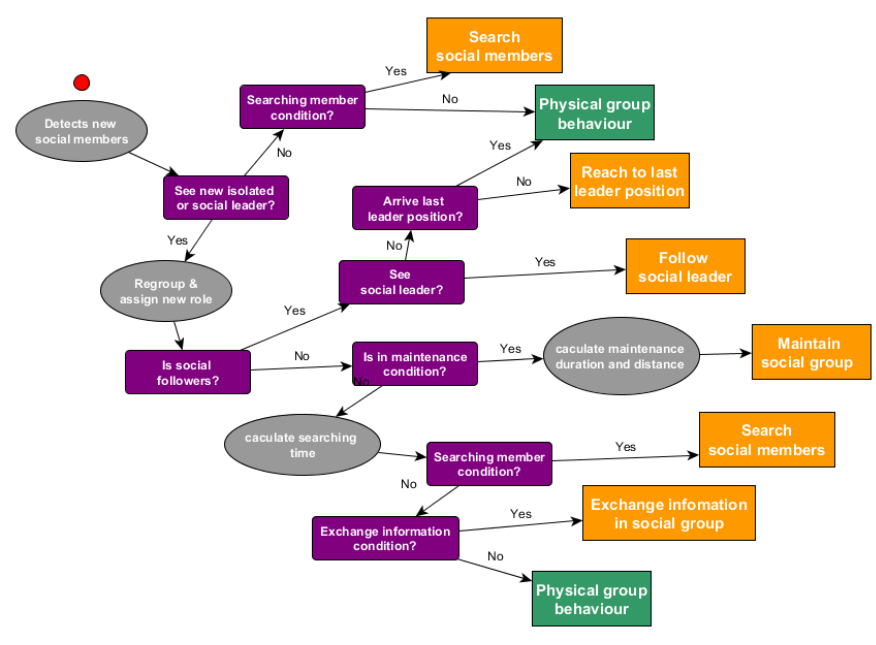

Fig. 16. Action decision-tree of agents in social group.

does not know this one.

4) Summary: The summary of all actions of agents in social group is presented by the decision-tree in Fig. 16.

We denote that in the detecting new social members process, we identify only isolated agents and social leader, because social followers do not influence on the regrouping and the assigning new role process. In addition, the social followers in old group are always the social followers in the new group. The social leader role only changes among the social leader and the new members (isolate member). With the influence of emotion, the social leader can become a social follower and inverse. The social followers can become isolated member if they lost their social leader. Social followers do not navigate by themselves, while isolated member and social leader must navigate by themselves to the exit. That means, isolated members and social leader can act and participate in the physical group or act as an individual but not for the social followers.

\section{Decision Making of Agent in Physical Group}

Individuals may have no social relationship with anyone, but in the evacuation process, they can find and regroup to create physical groups to help themselves moving and information exchanging. Isolated members and social leaders navigate as an individual, so they can participate (become the members) to the physical group. Agents in physical groups act almost similarly as agents in social groups. There exist actions such as following the leader, reaching to the last leader position, maintaining group and exchanging the information in group. But there does not exist the searching members process in the evacuation.

1) Physical follower: Physical followers follow their physical leader. Isolated members and social leaders can become physical followers. In case where the social leaders are physical followers, they keep the role of social leader, but they also have the role of physical leader to evacuate from the crisis. However, their first priority is the role of social leader. That means, they can leave from the physical group to maintaining

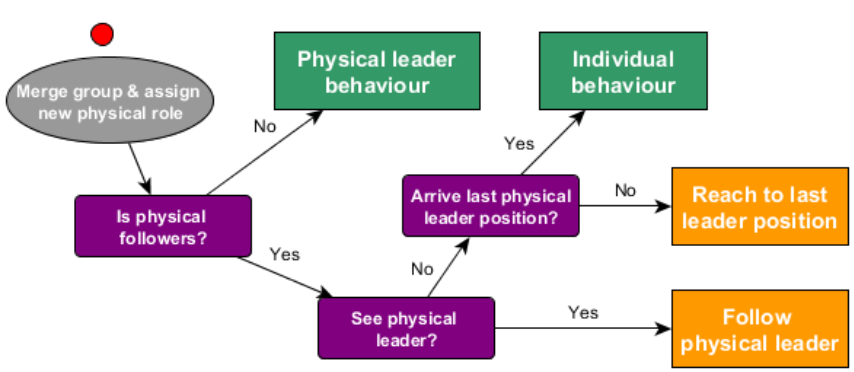

Fig. 17. Action decision-tree of physical follower agent.

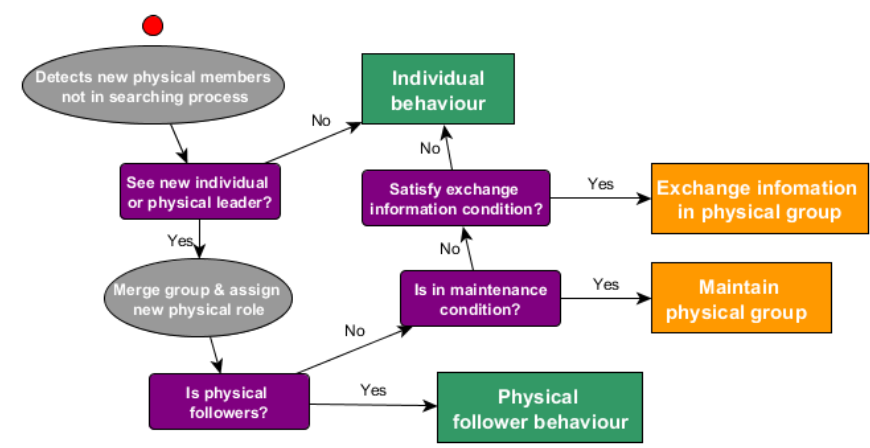

Fig. 18. Action decision-tree of physical leader agent.

their social physical group. The social followers in all cases always follow their social leader.

Isolated agents could take the physical follower role in a physical group, but if they see their social members, they could not become the members of this physical group (in this case they are the social followers).

Physical followers act as social followers such as reaching the last position of physical leader, following the physical leader. Caused by the weaker relation of physical group than that of social group, more physical followers could leave from the group (and act as an individual) than social followers. The action decision-tree of physical follower agents is presented in Fig. 17.

If physical followers follow a physical leader, they will continue to follow their leader and do not change to another one who is closer. in case of losing their physical leader, physical followers will try to reach to the last position of their leader before changing immediately when seeing another leader. So in fact, physical followers will change to the individual, and then, these individuals can join to another physical group.

2) Physical leader: Physical leader leads all physical followers to exit from the crisis. Isolated members and social leaders can become physical leaders. If social leaders become physical leaders, that means they take the two leader roles of both physical social group and physical group. So these leaders lead both social members and strangers.

The action decision-tree of physical leader agent is presented in Fig. 18. A physical group can extend if the physical 


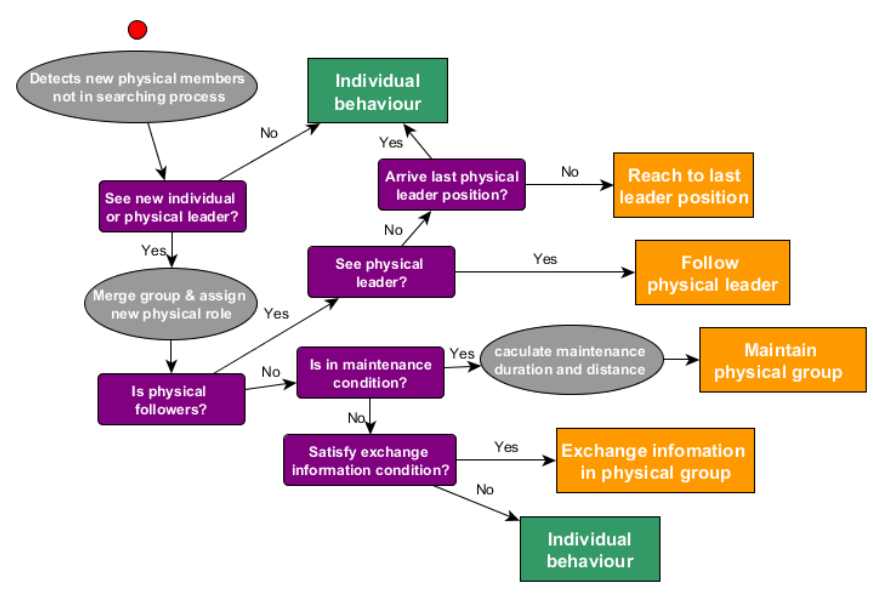

Fig. 19. Action decision-tree of agents in physical group.

leader sees other individuals and other physical groups. Isolated members and social leaders are considered as individuals if they are searching member action. They do not participate in any physical group. The physical leader do not do merging group action with these agents. After merging with the new members, the physical leaders continue navigating as individual (navigate by themselves) to exit. They can do the exchange information action with members in the physical group and maintenance group if the conditions for exchange information and maintenance are triggered.

Individuals, if they see each other, can create a new physical group. The individual with highest groupInfluence will become a physical leader, and the others become the physical followers.

3) Summary: The summary of all actions of agents in physical group is presented by the decision-tree in Fig. 19 .

\section{Decision Making of Individual Agent}

Individuals (who do not belong to any group), physical leaders, social leaders and isolated members navigate by themselves and we understand that they adopt individual behaviour. They do the actions that are represented by the decision-tree in Fig. 20.

Individuals when they do not see nor follow security agents, if they see exit doors they will choose the nearest one and reach to this exit door. When they arrive and this exit door is not a closed one, they will escape from the crisis area. If they know that this exit door is closed, they will update their knowledge and then continue navigating to another one. If there is no information about the exit doors, individuals can request the guidance from other persons. If there is someone who is ready to help, two persons will wait and exchange the information about the exit door. If individuals do not receive information from anyone, they may follow other persons (follow crowd), follow the wall or follow one direction to find the wall and after that finding an exit door while moving around the wall (we suppose that the exit doors are always located beside the wall). The detail of help by information

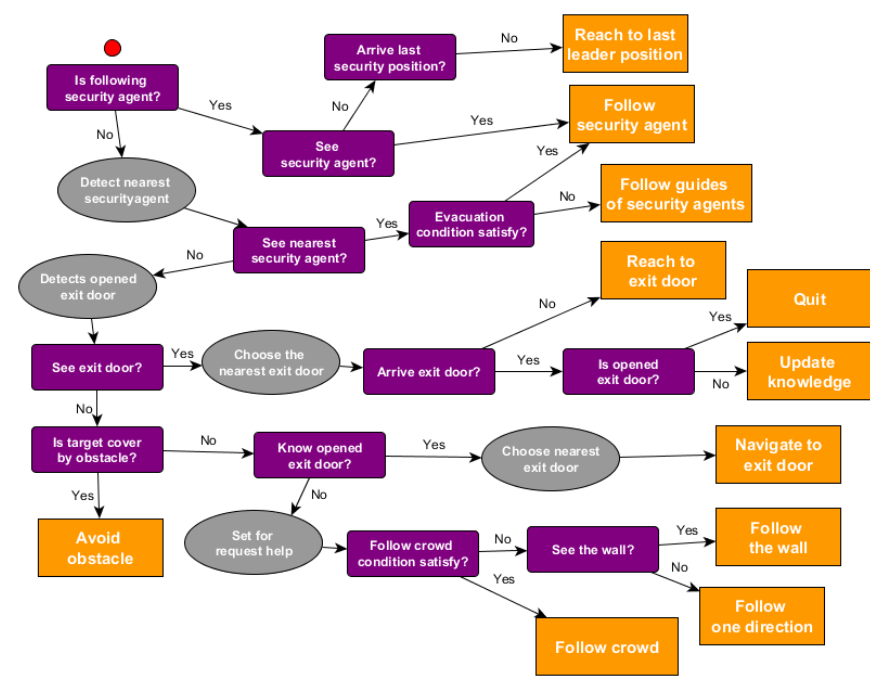

Fig. 20. Individual action decision-tree.

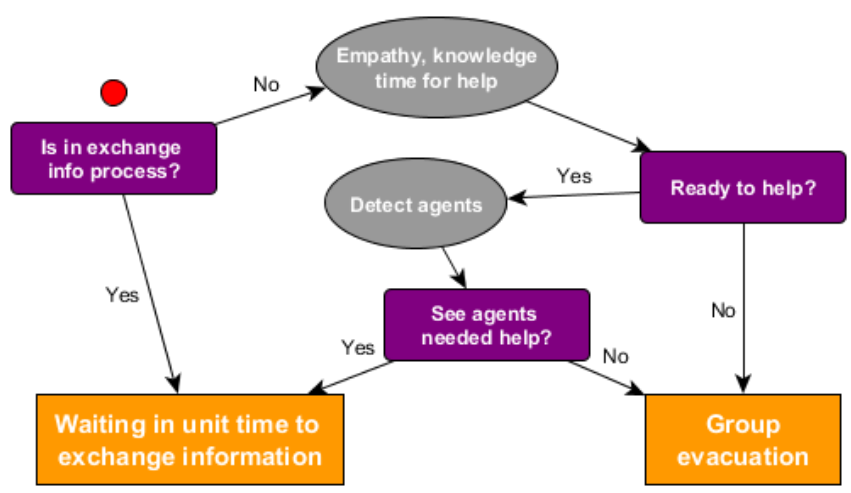

Fig. 21. Help by exchange information decision-tree.

exchanging action is represented by the decision-tree in Fig. 21.

In one step, agent can exchange the information with only one agent. Similarly to information exchanging in group, two agents have to spend a unitTimeExchange to exchange information about exit door. In each step of navigating to the target, if this target is covered by the obstacles, the individual will be triggered to take avoiding obstacle action.

\section{E. Decision Making of Security Agent}

Security agents take an important role in the evacuation process because they know all information about the place. Furthermore, they are trained for evacuation in case of crisis situation. They have the crisis experiences and also the knowledge and information about the customer, also the current situation of evacuation process. We suppose that security agents can do some actions such as guiding the way (indicating the best exit door with the lowest travelling time) for customers and participate directly in the evacuation process with the leader role. To simulate these two main actions, we suppose when crisis begins, security agents will stay in one place to 


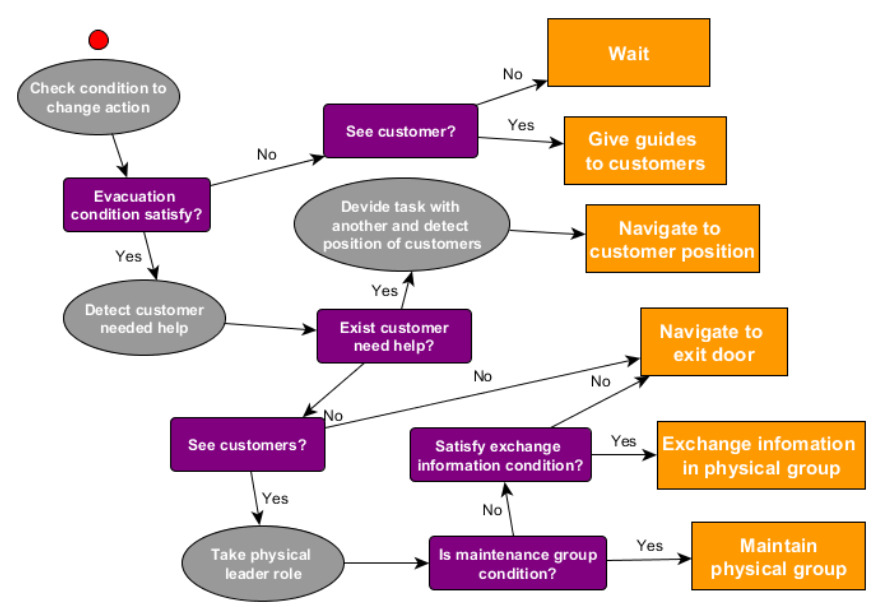

Fig. 22. Action decision-tree of security agents.

guiding customers by exchanging the information about exit doors. Security agents can trigger the evacuation action if the conditions of evacuation are satisfied. These conditions compose of the duration of crisis, the survivor rate of customer and the helping requirement of the customers. When customers needed help, security agents can navigate to their position. If there is no particular requirement from customers and if they do not see any customer, security agents will navigate to the exit door. If security agents see the customers, they will take the physical leader role and do the information exchanging action or group maintaining action. The detail of these actions of security agents are represented by the decision-tree in Fig. 22.

Security agent force all members in physical groups become their physical followers. Social followers always follow the social leader (who can become a physical follower with the physical leader is a security agent). We suppose that the priority of the relationship in social group is higher than that one of security agents. Security agents have particular actions that the physical leaders do not do such as going back to the location of people needed help from the exit door location. With the communication among security agents, they can share their tasks to command different customers.

\section{F. Influence of Emotion on Decision Making of Agents}

All the decision-tree actions above are related to group evacuation and do not taking into account yet the influence of emotion. But the emotion takes an important role in the decision making of agent in emergency evacuation. In this section, we will formalize the influence of emotion on the decision making of agents. To understand more clearly, we will present this influence on social group, physical group and individuals. Each state of emotion causes different influence on the decision making of agent. We use the state of emotion instead of emotion levels because the emotion level is a float value between $[0,1]$, and we can not estimate it because there are unlimited cases. While with states of emotion, we can have limited cases (we let four states only) to estimate the influence of emotion. The emotion level may be different but if the emotion state is the same, its influence on the decision

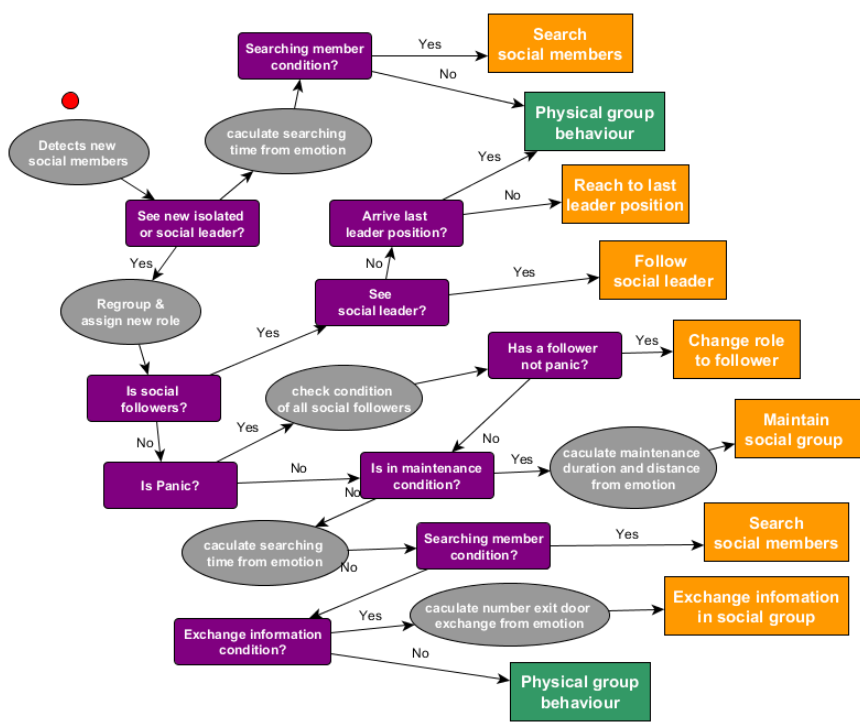

Fig. 23. Influence of emotion on action decision-tree of agents in the social group.

making is equal.

1) Influence of emotion on agents in the social group: Emotion can have influence on the time of doing some actions: the searching time, the waiting time, the distance (with followers) while doing maintaining action, the maximal exit door number needed to exchange in the exchanging information action (as we know that agents take a unitTimeExchange to exchange one exit door among all members in group). Emotion can also trigger new actions that are not existed in the group evacuation, such as: changing social leader role action if the social leader is in panic. The influence of emotion on the decision making of agents in a social group is represented in Fig. 23.

Higher state of fear level causes the lower searching time, the lower waiting time, the lower maximal exit door number exchange and causes the higher group maintaining distance. To define the maintaining group action, we need to know two distances: one is the distance to go back to the followers and the other is the distance to slow down the speed. With four states of emotion, we must have three thresholds for each above parameter. So with five parameters, we must create fifteen thresholds to manage the influence of emotion on the actions of agents in social group.

In case of panic, the searching time, the waiting time and maximal exit door number exchange are set to zero. That means panic agents do not searching other missing social members, do not waiting other members, and do not exchanging the information. If all members in a social physical group are in panic, the social leader of this group does not change. In this case, this leader does not decide to exchange the information nor wait other members in group. But if exist other members who are not in panic, the social leader role will change to the person who has the highest groupInfluence. And without the panic, this new leader can decide to wait social followers and exchange the information in group. The threshold of distance of going forward followers can be changed to equal to the 


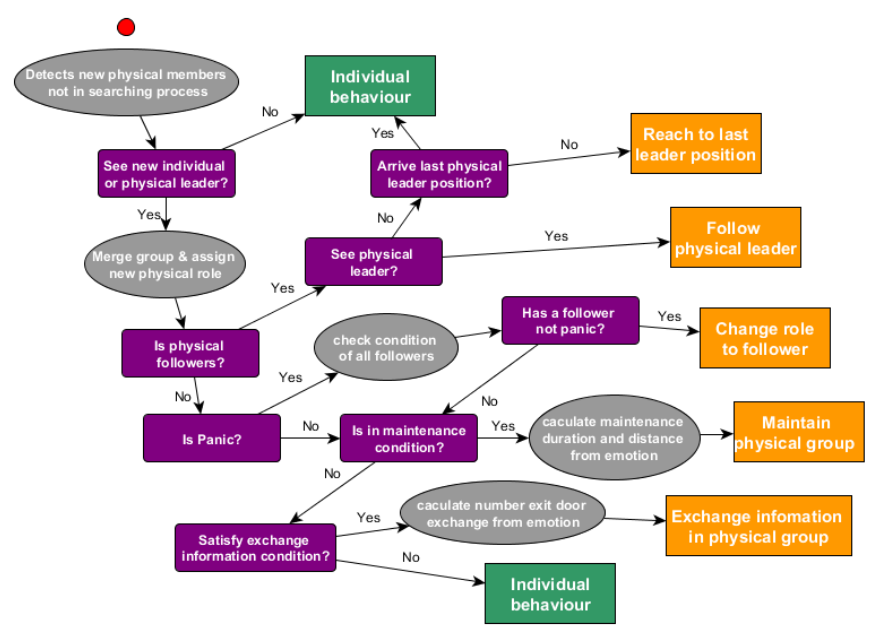

Fig. 24. Influence of emotion on action decision-tree of agents in the physical group.

visual radius globalRadius. Therefore, the social leader who is in panic, will not do this action and only do the slow down action.

With the stateFear state, social members take less time to waiting or searching members than with a stateAlittleFear state. And with stateNormal state the social agents act as normal agents. That means, emotion does not have influence on the actions of agent.

2) Influence of emotion on agents in the physical group: Members in physical groups act almost similarly to those in social physical groups. So, the influence of emotion on the physical members is almost similar with those on the social physical group. The influence of emotion on action of agents in the physical group is represented in Fig. 24.

Emotion has also influence on the actions of physical leader and physical followers. If a physical leader is in panic, his physical followers are checked if exist one physical follower who is not in panic and has the highest groupInfluence and this follower will become a new physical leader of this group. And the physical leader now becomes a physical follower of the new leader. Emotion has influences on: the waiting time of maintaining action, the distance triggering that action, the time of exchanging information in group. Note that, the relation of members in physical group is weaker than that in the social group, so the with the influence of emotion, these relations must be respected. That means, with the same emotion state, the waiting time of physical leader is less than that one of the social leader, and similarly with the time of exchanging information in group. The distance triggering maintaining action of physical leader is higher than that one of social leader.

3) Influence of emotion on individual agents: If agents navigate by themselves, we suppose that the emotion does not cause the losing of their mind. That means, the emotion does not have influence on the personality, and agents still remember the position of exit door if they know it before the crisis. With this hypothesis, if agents know exit doors, they have the capacity of navigating to these exit doors, even they

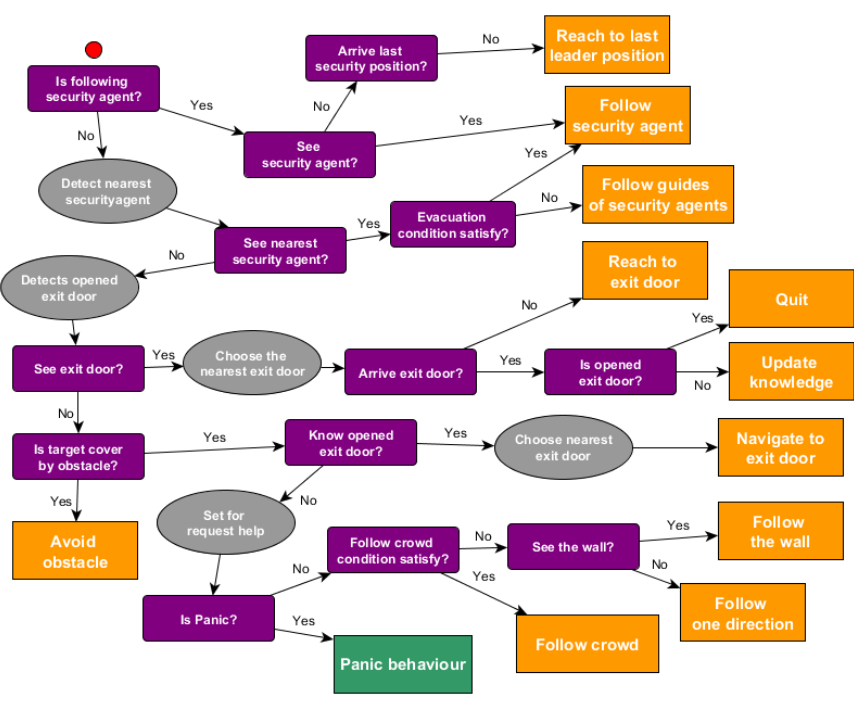

Fig. 25. Influence of emotion on action decision-tree of individual agents.

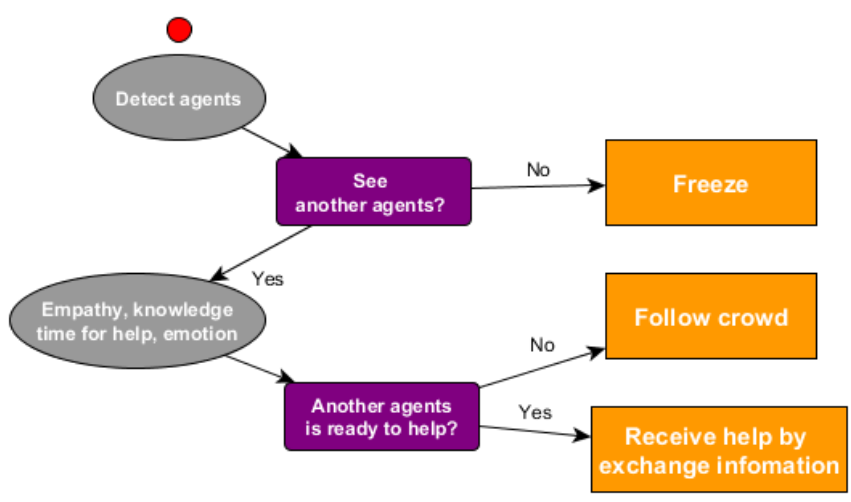

Fig. 26. Action decision-tree of panic agents.

are in panic state. But emotion has influences on the skill of choosing the best way to exit. With these analyses, we suppose if agents are in panic, they do not do these actions and react as with panic behaviour. The action decision-tree of individual agents is represented in Fig. 25.

And the action decision-tree of panic agents is presented in Fig. 26.

If panic agents do not see any other agents, they will be freeze. That means, they stay alone, do not move and can send the helping request to others. In case of seeing other agents, and these agents are ready for help, the panic agent will receive the helping information of exit door. If they do not receive any information, the panic agents will decide follow others to exit. That means, they do the following crowd action.

\section{Simulation: Implementation And Results}

\section{A. Implementation}

For the current works, we extended the existing simulation that was described in our previous works [46]. The following were added in this new version to taking into account the 


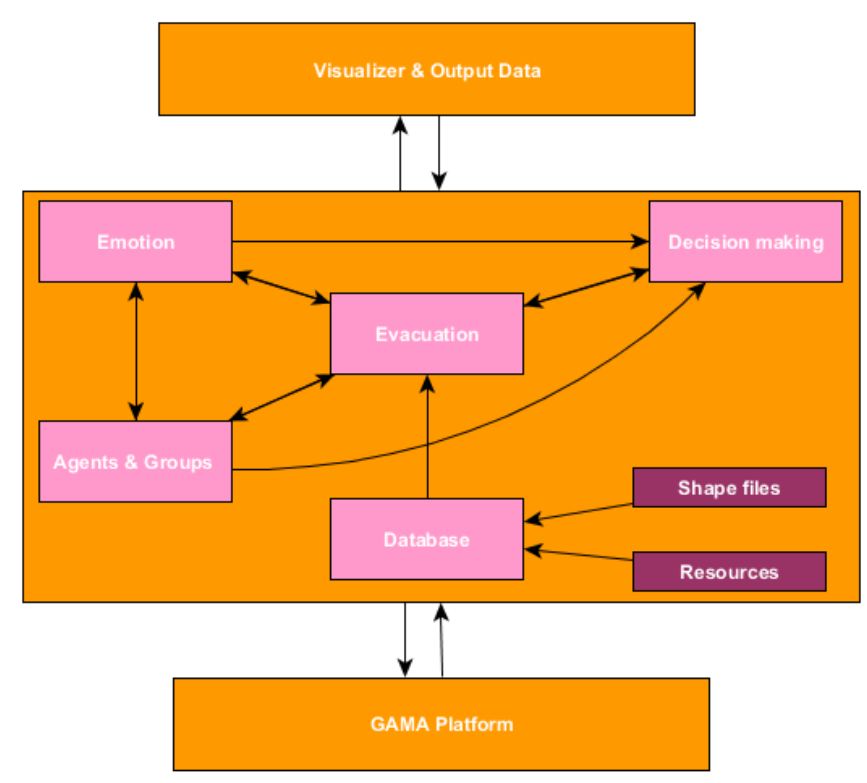

Fig. 27. Overall architecture of the simulation system.

effects of social relations and emotion on decision making, such as: 1) Complex actions relating to group such as maintaining group, searching missing members, exchanging the information, etc; 2) Emotion dynamics model.

The overall architecture of the simulation system is presented in Fig. 27. It has three layers: 1) Visualizer and Output Data layer presents the output and visualization of the simulation; 2) GAMA platform ( [47]) is an agent-based simulation platform with GIS integrated. All modules of the simulation are built with this platform; 3) Principal modules that are represented by the rectangles, and arrows are represented the impact of one module on another one.

The simulation environment is a super market with obstacles that are the shells, the walls around and the exit doors. The fires can burn only the shells and can propagate by time. The fires create the smoke and both of them can spread by time. The alarms are supposed to sound immediately when having the fires on the supper market, and people start evacuating at this moment. People can have the social relationship with the others before the crisis.

To simulate group behaviours, we need to control social and physical groups, and their members. First, with social group, we initialize the number social groups (socialGroupNumber) and the number of social members in each group. Each social group socialGroup $i$ has its identification idsocialGroup, and its all members have the same

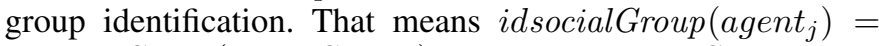

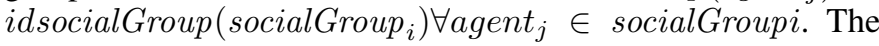
number of agents in all social groups must less than the number of customers. All agents has their own id are called idAgent.

Each agent is initialized with two variables relating to his roles in group idsocialGroup and idPhysicalGroup: idsocialGroup(SocialMembers) $\neq$ null and idsocialGroup(Individuals) $=$ null. idPhysicalGroup $=$ null for all agents in the initialization, but when they participate in a physical group, this value will be changed to equal to idAgent(PhysicalLeader). There are the lists of role of agent: \{isolateAgent, socialLeader, socialFollower \} for social groups, and \{individualAgent, physicalLeader, physicalFollower $\}$ for physical groups. Agents take the isolateAgent and socialLeader roles will be assigned automatically the individualAgent role if they do not participate in any physical group. The physical role can be changed to socialLeader or socialFollower if agents are members of physical group. Agents with socialFollower role do not have the physical role because they always follow socialLeader, and cannot participate in any physical group. Note that, the socialLeader and the physicalLeader are created dynamically depending on the evacuation of all agents, their attributes and emotions.

Agent's location is placed randomly in the moving space of super market. Agents in a socialGroup can be initialized closely or far away. If they are far away, we suppose that existing a meeting point where all agents in the socialGroup will reach to it in a searchingTime. This point can be the center location of all agents, or the position that all agents think the others will go there when a trouble happens. A socialPhysicalGroup who is leaded by the social leader, navigates to the exit door if there is no missing member or has no time to searching others. The socialLeader will lead this group and all socialFollower follow him. The socialLeader and the isolateAgent in the evacuation process can participate in a physicalGroup. And the physicalLeader of this group leads all physicalFollower to the exit door. The physicalLeader and individualAgent can navigate by themselves or follow other agents (other followers of other groups) to exit door.

The emotion of an agent is influenced by the environment, his attributes, and his neighbours. Agents in the same socialGroup have more impact than the strangers. So we can set the coeffNeighbour of social members higher than that one of stranger. There are four states of emotion: \{stateNormal, stateAlittleFear, stateFear, statePanic\}. With the stateNormal state, agents act as normal (there is no impact of emotion on their actions). With statePanic state, agents do not search missing members, do not waiting other members, and do not do exchanging information or guidance information to others. The socialLeader and physicalLeader roles may be changed to the one of a follower who is not in panic. Panic agents can be freeze if they do not have the knowledge of the exit door. The stateAlittleFear and stateFear will influence on the time of doing some above actions, but they do not make the agent freeze or change their roles.

1) Emotional group evacuation: The emotion of agent is calculated in each step of evacuation. It influences directly the decision of agent and therefore impacts on the group evacuation process.

In order to calculate fear intensity, we first calculate the emotion decay, then the impact of environment, and last the influence of neighbours. After having this value, we set the state of agent. Based on the emotion state, we calculate the following values: waitingTime, exchangeTime, maintainDistance, waitingDistance, gobackDistance (for both social group and physical group), searchingTime (only for social group). In 
panic state, waitingTime, exchangeTime, searchingTime are set to zero.

If agents decide to give (receive) guidance information to (from) the others, or to exchange information in the groups (that means they have time to do this action, so these agents are not in panic state), they will lose time to do these actions. In simulation, we present these actions by the waiting function. The flagExchangeInfo attribute of agent will be set to true while agent doing these actions.

If agents are in the exchange information process, they cannot do any other action. They must wait until this process finished (except the case for panic agents). After waiting to exchange the information, the speed of agent will be restored and continue the evacuation process.

After managing the exchange information process, we must to control the group evacuation. The social group behaviour has the highest priority, after that are the crowd behaviour or the physical group behaviour, and last is the individual behaviour 11

We create some variables to easily control (enable or disable) the influence of these behaviours. We can create many cases with enable or visible one or many behaviour to evaluate and compare their results. The detail of each algorithm according to each behaviour will be presented in the next sections.

2) Agents in social group: As the socialFollower does not change his role in the expanding group process (he may change his role only in case of existing the panic social leader in his group). So there exist only isolateAgent and socialLeader who decide this action. Each time having a new member, he needs to compare with the socialLeader of this group to choose which one will become a new socialLeader.

If a socialLeader is in panic, he changes his role to a social follower who is not in panic, or does not change his role in case all his members are in panic too. A socialLeader checks to do maintaining action or not in each step. The priority of this action is higher than that of the searching missing member action, because we suppose in the searching missing members process, a socialLeader still maintains his group. If a socialLeader is in the searching time, he explores the space to find his members. If not, he may request to exchange information with his followers or follow the crowd or physical group behaviour.

The isolateAgent searches missing member if he is in the searching condition. If not he adopts the crowd behaviour or physical group behaviour.

The socialFollower always checks the visibility of his socialLeader to follow. If it is not visible, the socialFollower try to reach to its last position before changing his role to the isolateAgent. If he changes the role, we must remove him from the social follower list of the socialLeader.

3) Agents in physical group: There are three roles relating the physical group: socialLeader, physicalFollower, individualAgent. There is no searching missing member action in physical group, but the order of other actions are almost similar to the social group.
The physicalLeader in panic state may changes its role to physicalFollower. Without this state, it maintains its physical group or follows the individual behaviour. The physicalFollower acts the same actions as the socialFollower. If leaving from the physical group, the physicalFollower will change its role to individualAgent.

4) Individual agents: The actions of individual agent are almost similar to the customer's actions in the first simulation. We improve these old ones by adding the influence of emotion and following guidance from security agents. We also add the freezing behaviour in case of panic state and the following crowd actions. We keep other actions such as reaching to the exit door, following security agents, reaching to the last position of security agent, choosing and navigating to the known exit door, following the wall, following one direction, avoiding obstacles.

The individualAgent stays while receiving the guidance information from security agents. This process is similar to the receiving guidance from other customers (in case of panic or has no information of exit door). Therefore, panic individuals can follow other agents (follow crowd) to exit.

5) Security agents: In this version of simulation, we improve the behavior of security agent by adding the guidance information action and improve the evacuation action. Now, the security agents can stay in one place to provide the best exit door to customers (it may different with the nearest exit door). And for evacuation action, security agents can automatically detect and go back to the customer's position to help.

We distinct two actions by using the function getMissionSecurityAgent(). For now, similarly to some actions as searching members or waiting members, we suppose in first period, security agents provide guidance information to customers in the waitingTimeGuide. Then, if the simulation time passes this duration, they start doing the evacuation process by leading physical groups to the best exit door, or going to the customer's position to help. With the communication among all security agents, they can share their tasks to take care each customer without duplication. That means, there is no case that two security agents help one customer while existing another one who needs help.

\section{B. Results and Evaluation}

\section{1) Real-time results of emotional group evacuation:}

a) Social, physical group evacuation: We create three social groups with the same number of members. Each social group has different color (red, blue, pink). Individuals are the black agents with no social relationship with other agents. We suppose all agents have the same visual radius.

Every agent starts evacuating when hearing the alarm even if they do not see the fire. Non panic agents in social groups will search the missing members in their social group (Fig. 28). If agents see the fire, their emotion are triggered, and may influence their decision making. We set a simple searching missing member mechanism so that all agents in the same social group go to the meeting location (perhaps the center of all agents in group), while the black individual agents navigate immediately to the exit door. The social agents in searching process do not join to physical groups. But, the social members 


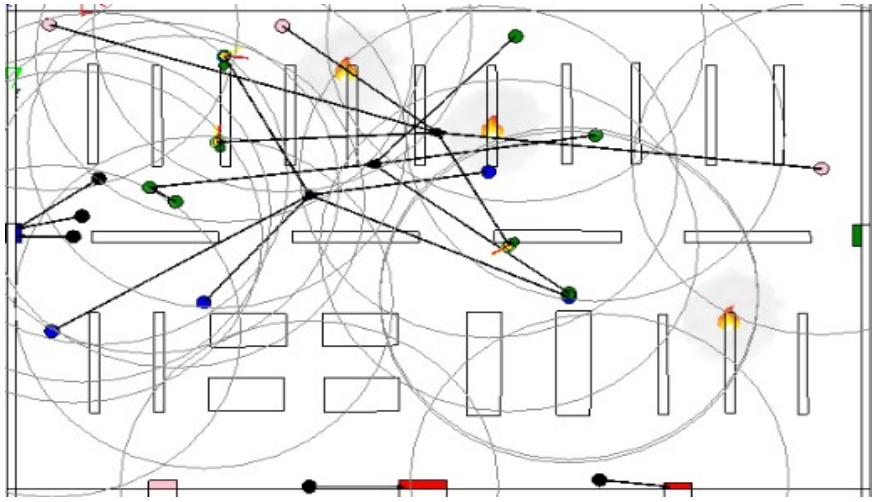

Fig. 28. Searching missing members.

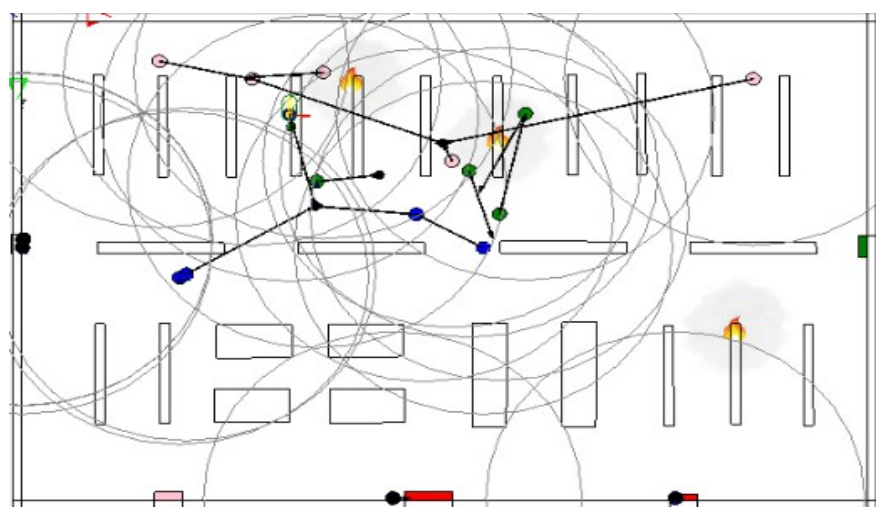

Fig. 29. Creating a social physical group.

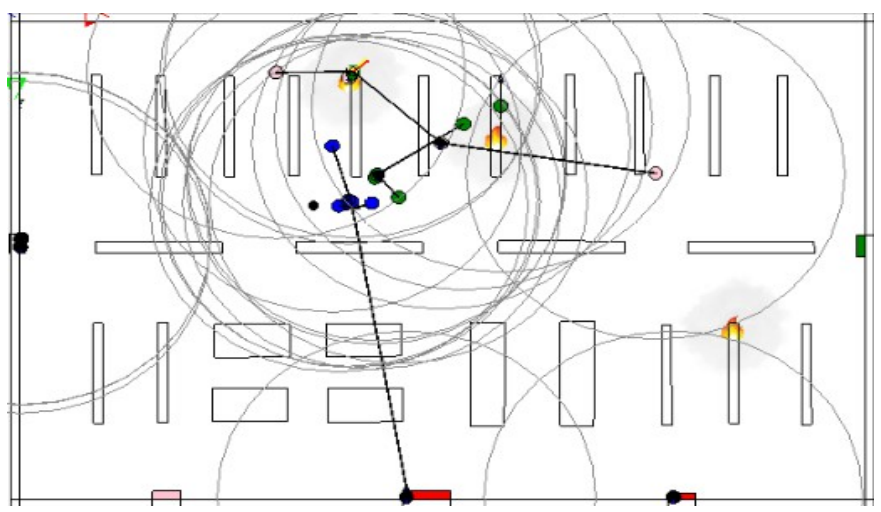

Fig. 30. Full member group evacuates.

can create a social physical group as Fig. 29. We see two social physical groups with blue agents. The reds agents also create the social physical group while moving to the meeting location. In Fig. 30, the blue social group has all its members, so all members start to navigate to the exit door.

For the physical group evacuation, we focus only on the back agents.

If individuals see the exit doors, they will reach immediately to the closest exit door. If they do not see the exit door, they can make the physical group (black agents on top of Fig. 31 ). We can see the physical leader of this group does not

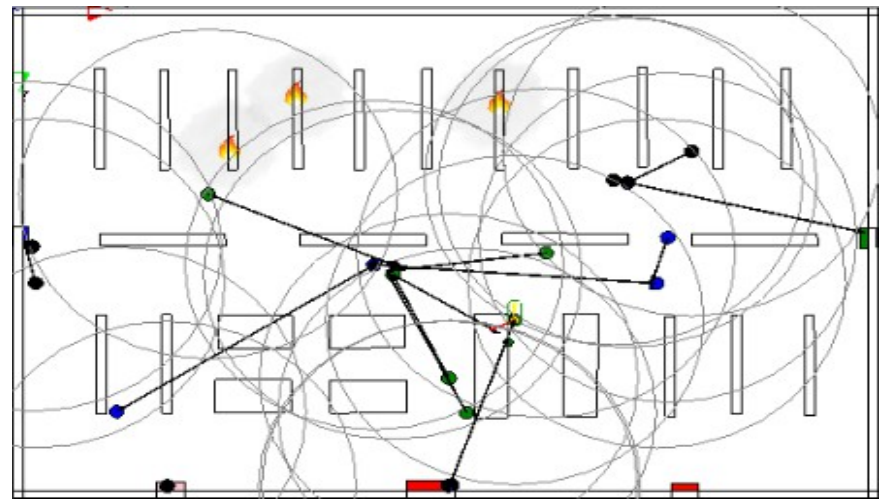

Fig. 31. Making physical group.

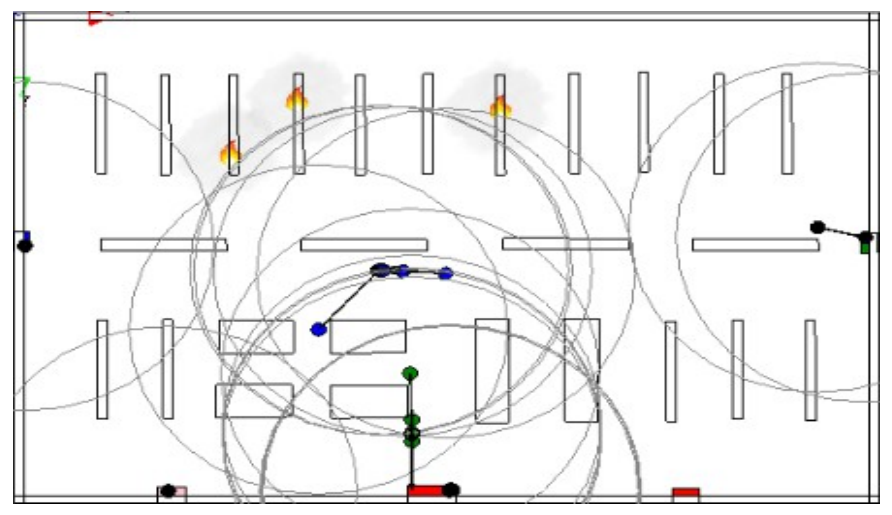

Fig. 32. Evacuation of social agents and individual agents.

know any exit door, and navigates following one direction. But with the exchanging information action among members in the group, the leader knows the nearest exit door and navigates to this door. We can observe, even individual agents quit from the supermarket, the social agents are still evacuated because they waste time in searching missing members (Fig. 32).

b) Emotional group observed: We calculate the fear level of agents and display these values real-time when agents move. We make only two groups (red and blue) with four agents for each group, and two individual agents to observe easily the changing of emotion.

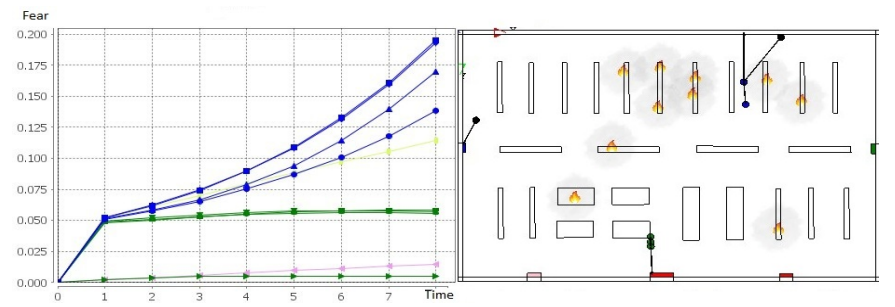

Fig. 33. Fear level changes after seeing the fire.

We can see these changes of all agents in the evacuation process. The blue agents have the higher fear level than that of the red agents, because they are closer to the fires (Fig. $33)$. The red agents detect the exit door and navigate to there, while the blue agents do not know any exit door, so they search 


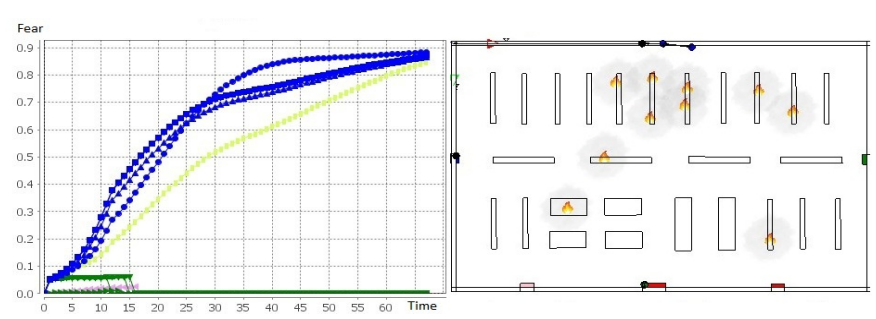

Fig. 34. Fear level increases when agents are closer to the fire.

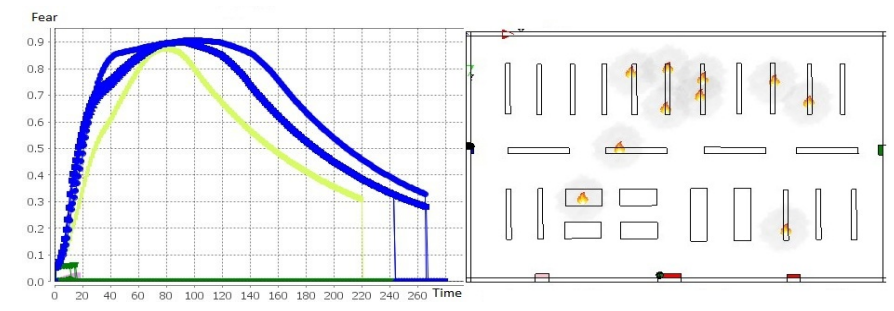

Fig. 35. Fear level decreases when agent far from the fire and dropped when agent escaped.

and then follow the wall to exit. The social leader of the blue group creates a physical group with other individuals (Fig. 33). The emotion of this agent is represented by the yellow line. This value increases quickly too. The emotion of other agents continue increasing because of the influence of the fires (Fig. 34). And if they move far from the fires, their emotion decreases. If they go out, their emotions are equal to zero (Fig. $35)$.

2) Impact of knowledge, exchange information in group and guide information among individuals on the survivor rate: We make the simulation's conditions are almost similar to the ones in the section above. But, in addition we change the following parameters: the social group behaviour, physical group behaviour, the helping behaviour (guidance information for stranger and exchange information in group), the rate of knowledge. The simulation time and others parameters are fixed. With each case, we run with the simulationTimes $=5$, and then calculate the average value. The value of other parameters are customerNumber $=50$, socialGroupNumber $=$ 6, socialMemberNumberInGroup = 4, socialMemberNearby $=$ true, simulationStep $=150$, rateKnowledge $=0.2$. There are five doors of the super market. And we suppose if agents' knowledge is set to true, that means they know two exit doors position and one of exit door is the main entrance, unitTimeExchange $=12$ (it takes 12 steps of simulation to exchange the information about one exit door), exchangeTimeTotal $=1000$ (we unlimited the exchange information action, so set the high value for this value). We disable the influence of emotion, calculate the survivor rate (Number of agents who arrive the exit door divided by the number of customer), and compare these values between the test cases in Table II. The results are presented in Fig. 36.

We see, in case of enable social behaviour or physical behaviour (case 1,2,3), the survivor rate in case of enable exchange information and guidance information are similar or
TABLE II. TEST CASES WITH GRoup BehaVIOUR

\begin{tabular}{|l||c|c|c|c|}
\hline Test cases & 1 & 2 & 3 & 4 \\
\hline socialGroupBehaviorEnable & $\mathrm{T}$ & $\mathrm{T}$ & $\perp$ & $\perp$ \\
\hline physicalGroupBehaviourEnable & $\mathrm{T}$ & $\perp$ & $\mathrm{T}$ & $\perp$ \\
\hline
\end{tabular}

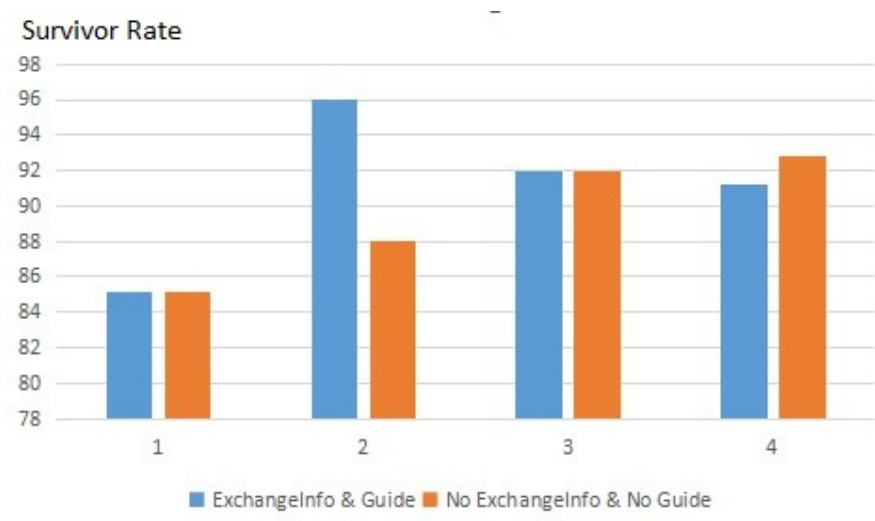

Fig. 36. Relation between helping, exchange information in group with social behaviour, physical behaviour, emotion.

better than that in case disable exchange information (the blue columns are higher than the reds ones). But it's not similar with the case of disable both social behaviour or physical behaviour. This phenomenon can be explained by the wasting time when guidance information among individuals in group is not effective. The individuals will delay the evacuation. Note that individual agents can guide any other agent who needed help if they have time. We set exchangeTimeTotal $=$ 1000 , so we can understand that agents always have time to support others. The exchange information among members in group is a little different. Leaders only decide to exchange information if their knowledge is different with that of all followers. The best result is in the case of enable exchange information and socialGroupBehaviorEnable = true, physicalGroupBehaviourEnable = false (the blue column in the second case). Because of socialMemberNearby $=$ true, so all social members of each social group are situated close. Therefore, with exchanging information action, the rate of knowing exit doors of social leaders is higher, and they can lead the social physical group exit safety. The result in case of disable exchange information, nor evacuation in group is better than the others (except the best result above). Therefore, the exchange information in group or the group behaviour can cause the delay evacuation, and then reduce the survivor rate.

We continue conducting more test cases by increasing the rateKnowledge $=0.8$ while keep all others parameters similar to the above test cases. Then, we compare the results between these test cases. Now, we have eight cases to compare that are presented in Table III. The results are presented in Fig. 37.

TABLE III. TEST CASES OF EXCHANGE INFORMATION AND GUIDE INFORMATION BY CHANGING rateKnowledge AND GROUP BEHAVIOUR

\begin{tabular}{|l||c|c|c|c|c|c|c|c|}
\hline Test cases & 1 & 2 & 3 & 4 & 5 & 6 & 7 & 8 \\
\hline rate knowledge & 0.2 & 0.2 & 0.2 & 0.2 & 0.8 & 0.8 & 0.8 & 0.8 \\
\hline social group & $\mathrm{T}$ & $\mathrm{T}$ & $\perp$ & $\perp$ & $\mathrm{T}$ & $\mathrm{T}$ & $\perp$ & $\perp$ \\
\hline physical group & $\mathrm{T}$ & $\perp$ & $\mathrm{T}$ & $\perp$ & $\mathrm{T}$ & $\perp$ & $\mathrm{T}$ & $\perp$ \\
\hline
\end{tabular}




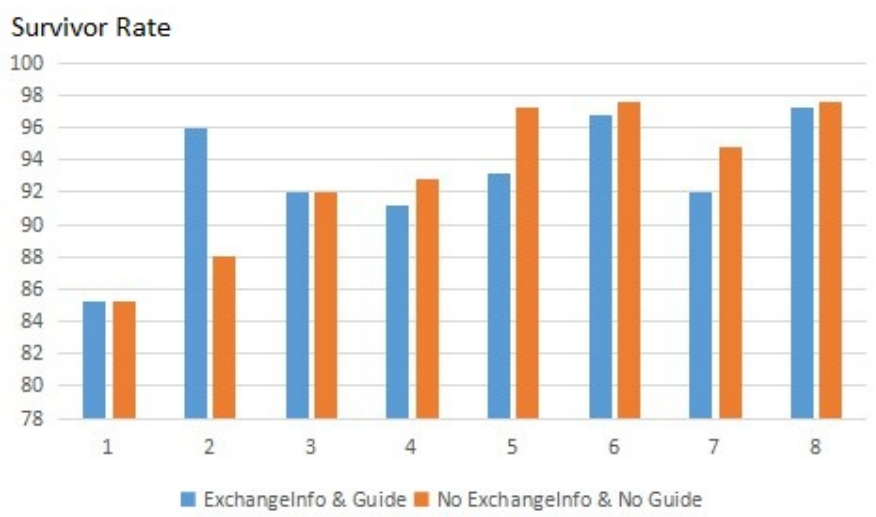

Fig. 37. Relation between helping, exchange information in group with social behaviour, physical behaviour, emotion in case modify the rateKnowledge.

We can observe that the survivorRate is higher if the rateKnowledge is high (the columns in case from 4 to 8 are higher than the ones from 1 to 4 ). The interesting thing is with the higher rateKnowledge, the exchange information and guide information always cause the lower survivor Rate (the blue columns are lower than the red columns from 5 to 8 ). And without group behaviour, the survivor rate are highest (columns of case 8 are highest). That means both the exchange information and the group behaviour are not effective for the group who has high knowledge of exit door.

We continue discovery the exchange information in group by run other test cases with and without influence of emotion. We also test for the case of making the positive impact of emotion or not such as increasing the speed of agent if fear state increases. These test cases are presented in the Table IV.

TABLE IV. TEST CASES OF EXChANGE INFORMATION AND Guide INFORMATION BY CHANGING EMOTION AND GROUP BEHAVIOUR

\begin{tabular}{|l||c|c|c|c|c|c|c|c|}
\hline Test cases & 1 & 2 & 3 & 4 & 5 & 6 & 7 & 8 \\
\hline emotional & $\mathrm{T}$ & $\mathrm{T}$ & $\mathrm{T}$ & $\top$ & $\perp$ & $\perp$ & $\perp$ & $\perp$ \\
\hline social group & $\mathrm{T}$ & $\mathrm{T}$ & $\perp$ & $\perp$ & $\mathrm{T}$ & $\mathrm{T}$ & $\perp$ & $\perp$ \\
\hline physical group & $\mathrm{T}$ & $\perp$ & $\mathrm{T}$ & $\perp$ & $\mathrm{T}$ & $\perp$ & $\mathrm{T}$ & $\perp$ \\
\hline
\end{tabular}

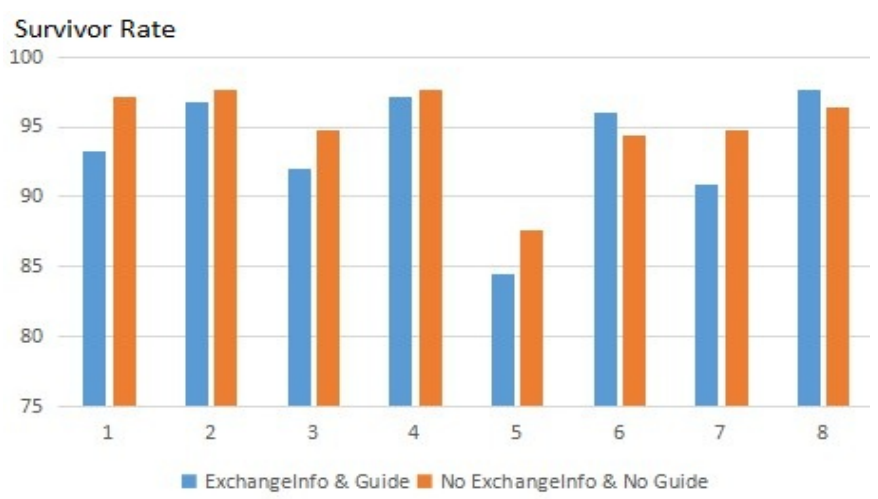

Fig. 38. In case emotion causes increasing agent speed.

With the increasing speed according to the emotion state, the survivor rate in case of enable emotion is higher than that of disable emotion impact (the columns from 1 to 4 in Fig. 38 are higher than these ones from 5 to 8). But without increasing

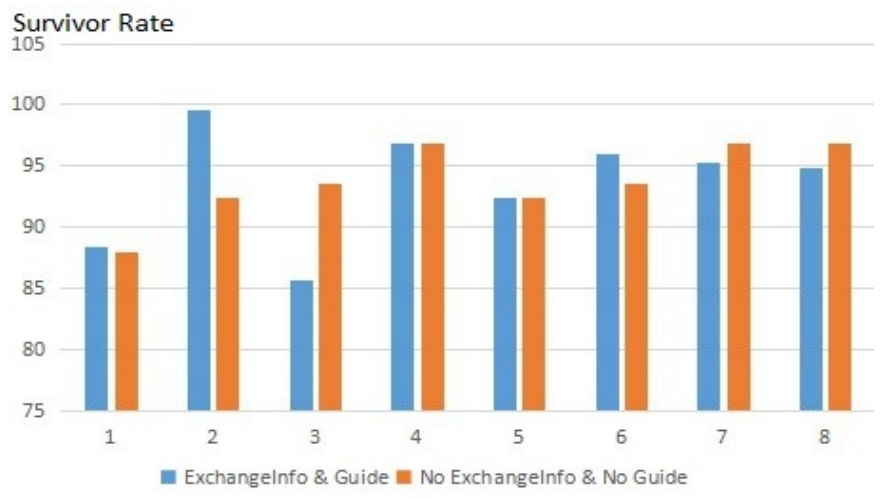

Fig. 39. In case emotion does not cause changing agent speed.

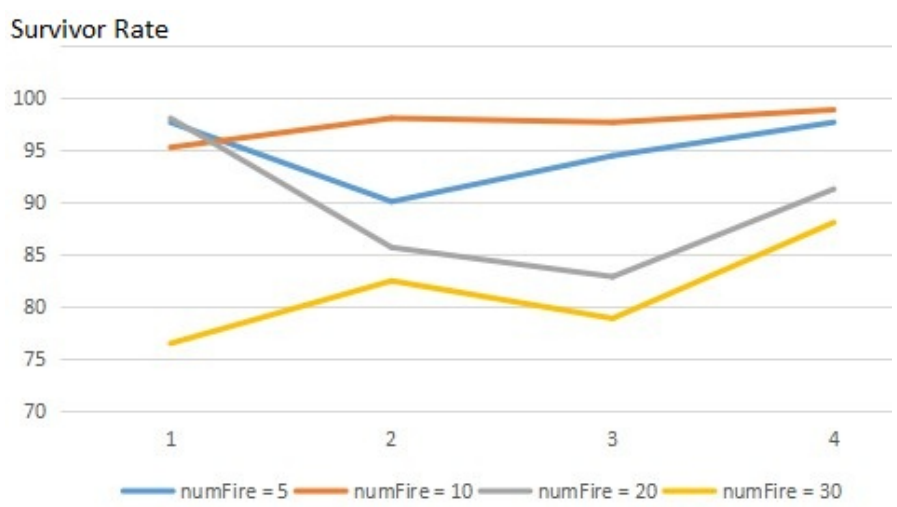

Fig. 40. Influence of emotion, group behaviour, fire number on survivor rate.

speed, the results are not clear in case of enable or disable emotion impact (Fig. 39).

3) Impact of fire number, emotion and group evacuation on the survivor rate: We conduct simulations with these parameters: customer Number $=50$, socialGroupNumber $=6$, socialMember NumberInGroup $=4$, simulationStep $=$ 200 , rateKnowledge $=0.2$, unitTimeExchange $=$ 12, exchangeInfoInGroupEnable = true, guideInformationEnable $=$ true. We change emotionalBehaviourEnable, socialGroupBehaviorEnable, physicalGroupBehaviourEnable among [true, false]. We also run each case of simulation five times, then calculate the average values. We compare the survivor rates between the test cases (in Table II). And the results are presented in Fig. 40 .

We continue testing with the cases of enable and disable impact of emotion. The comparison results are presented in Fig. 41 with enable emotion case (columns in 1, 2, 3, 4) and disable emotion case (columns in 5, 6, 7, 8).

In case fireNumber $=10$ (red columns), the survivor rate in case of enable emotion is higher than disable emotion (red columns from 1 to 4 higher than from 5 to 8 ). The blue columns in case of enable emotion is more positive than disable emotion (case 1, 3, 4 are better than 5, 7, 8, except case 2 is worse than 6). With increasing the fireNumber $=20$ and fireNumber $=30$, all cases without impact of emotion are better than with impact of emotion. These phenomenons are 


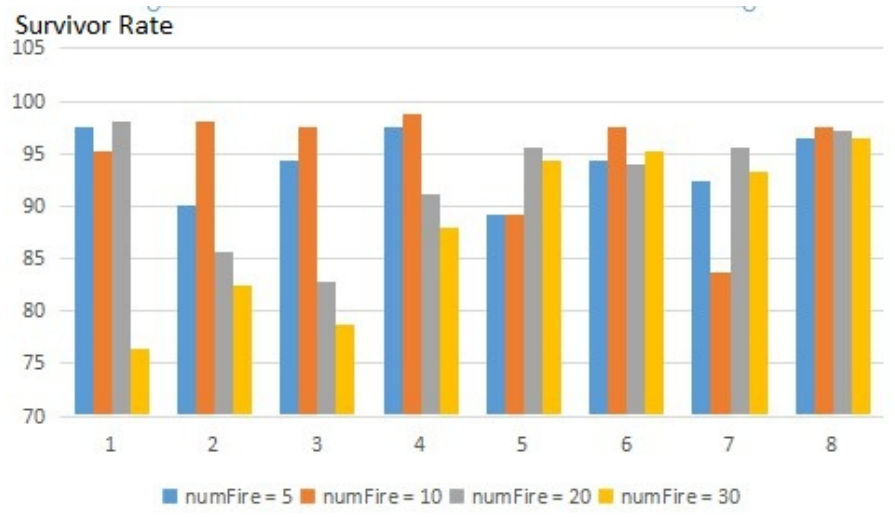

Fig. 41. Compare influences of emotion, group behaviour, fire number on survivor rate in case enable and disable emotion behaviour.

similar with the above test cases.

4) Impact of searching missing members, emotion, group evacuation on the survivor rate: To test the effective of searching missing members action, we change the searching time and the distance among the social members in the initialization. So we change searchingTime $=[20,50,80,120]$ (social agents search missing members only on this time, and then start navigating to the exit doors) and socialMemberNearby among [true, false], while keeping the other parameters: customerNumber $=50$, socialGroupNumber $=6$, socialMemberNumberInGroup $=4$, simulationStep $=$ 200 (the simulation runs only 200 steps and then stop), rateKnowledge $=0.2$, unitTimeExchange $=$ 12, exchangeInfoInGroupEnable = true, guideIn formation Enable $=$ true.

To evaluate the influence of searching time and distance between the social members, we change searchingTime among [20, 50, 80, 120], and socialMember Nearby among [true, false], physicalGroupBehaviourEnable among [true, false], while keeping socialGroupBehaviorEnable = true and emotionalBehaviourEnable $=$ false. That mean we do not calculate the influence of emotion on this test case. We run each test case 10 times, and then calculate the average values. The results are presented in Fig. 42. Four left values accord to physicalGroupBehaviourEnable = true, while four right values accord to physicalGroupBehaviourEnable $=$ false.

We easily observe if all social members are close when the evacuation starts, the survivor rate is always higher (the red columns is higher than blue columns) because they don't waste the time to search missing members and can navigate immediately. The red columns doesn't depend on the searching time. While the blue columns depends on these values. With increasing searching time, the survivor rate decreases (except the case searchingTime $=120$ and physicalGroupBehaviourEnable $=$ true). The survivor rate in case disable physical group behaviour is higher than enable one (the 4 right columns are higher than the 4 left columns). That means, the physical group behaviour badly affect the survivor rate. This phenomenon is caused by the exchange information in physical group and some maintenance actions in physical groups which constitute the delay evacuation.

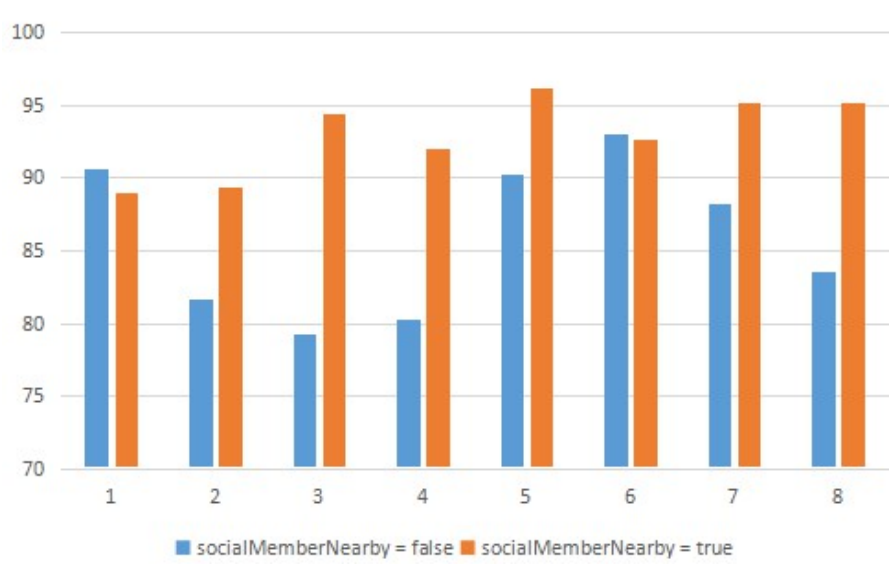

Fig. 42. Relation between searchingTime, socialMember Nearby, physicalGroupBehaviourEnable on survivor rate while fix , socialGroupBehaviorEnable $=$ true and emotionalBehaviourEnable = false.

\section{Survivor Rate}

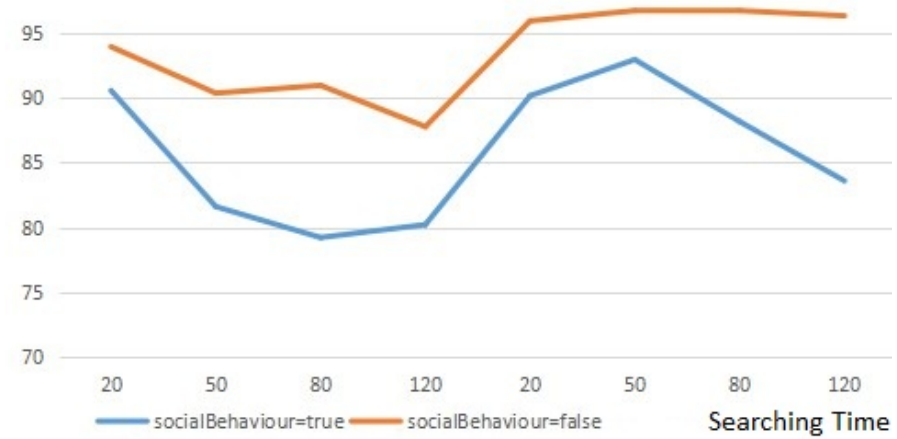

Fig. 43. Relation between searchingTime, socialGroupBehaviorEnable, physicalGroupBehaviourEnable on survivor rate while fixed, emotionalBehaviourEnable = false, socialMember Nearby $=$ false.

We continue to test case with enable and disable social group behaviour with the same parameters as above. The results are presented in Fig. 43.

The results without social group behaviour are better. That means if all agents in social groups don't follow the social actions such as searching missing members, regroup, or making social physical group, the survivor rate is higher. The best results is in case socialGroupBehaviorEnable $=$ false and physicalGroupBehaviourEnable = false (the right orange lines are highest). That means without both social and physical behaviour, the survivor rate is highest.

Now we test with the influence of emotion by comparing with case of enable emotion behaviour. All other parameters are same as above. The results are presented in Fig. 44.

With emotion impact in this case, the survivor rate is higher. The different survivor rate in case physicalGroupBehaviourEnable $=$ false is higher than in case physicalGroupBehaviourEnable = true (the distance between the right lines are higher than that one 


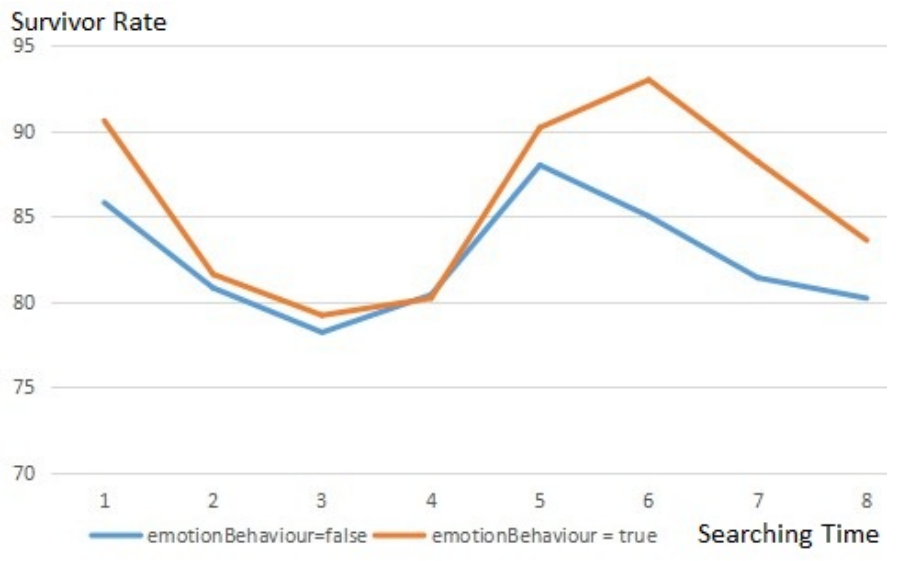

Fig. $44 . \quad$ Relation between searchingTime, emotionalBehaviourEnable, physicalGroupBehaviourEnable on survivor rate while fix socialGroupBehaviorEnable = true, socialMemberNearby $=$ false.

between the left lines).

\section{CONCLUSION}

In this paper, we firstly presented a formalization of group behaviors and actions in emergency situations based on social theory. By taking into account the social relations, it defined different types of people group, group attributes, group behavior and actions according to group's type. Secondly, we detailed the modeling of the effects of emotion and group behavior on occupant decision making in evacuation. Based on these two works, we extended an existing agent-based simulation of fire evacuation. Using the simulation, we conducted different experiments that allows to study the effect of group (with or without social relations) and emotion on the decision making in the case of fire evacuation in a supermarket.

Modeling different kinds of group, testing different strategies of fire evacuation for group are some of the perspective issues for our research in the near future.

\section{ACKNOWLEDGMENT}

This work is funded by the research project at Vietnam National University in Hanoi, number QG.15.31, on the modeling and simulation of fire evacuation in public buildings.

\section{REFERENCES}

[1] M. H. Nguyen, T. V. Ho, and J.-D. Zucker, "Integration of smoke effect and blind evacuation strategy (SEBES) within fire evacuation simulation," Simulation Modelling Practice and Theory, vol. 36, pp. 44-59, 2013.

[2] X. H. Ta, B. Gaudou, D. Longin, and T. V. Ho, "Emotional contagion model for group evacuation simulation," Informatica, vol. 41, pp. 169182, 2017.

[3] F. Qiu and X. Hu, "Modeling group structures in pedestrian crowd simulation," Simulation Modelling Practice and Theory, vol. 18, no. 2, pp. 190-205, 2010

[4] C. Mei Ling and L. Kincho, "Computational framework incorporating human behaviors for egress simulations," American Society of Civil Engineers, 2013.

[5] C. Mei Ling, P. Paolo, L. Jean-Claude, and L. Kincho H., "Simulating effects of signage, groups, and crowds on emergent evacuation patterns," Springer-Verlag London, 2014.
[6] G. Santos and B. E. Aguirre, "A critical review of emergency evacuation simulation models," Building Occupant Movement During Fire Emergencies Conference, 2004.

[7] M. L. Chu, P. Parigi, J.-C. Latombe, and K. H. Law, "SAFEgress: a flexible platform to study the effect of human and social behaviors on egress performance," SimAUD 2014, 2014.

[8] S. Okazaki and S. Matsushita, "A study of simulation model for pedestrian movement with evacuation and queuing," in International Conference on Engineering for Crowd Safety, vol. 271, 1993.

[9] M. Anthony R, "Understanding mass panic and other collective responses to threat and disaster," Psychiatry: Interpersonal and Biological Processes, pp. 95-113, 2005.

[10] J. Drury, C. Cocking, and S. Reicher, "Everyone for themselves? a comparative study of crowd solidarity among emergency survivors," British Journal of Social Psychology, vol. 48, pp. 487-506, 2009.

[11] B. Latane and J. Rodin, "A lady in distress: Inhibiting effects of friends and strangers on bystander intervention," Journal of Experimental Social Psychology, vol. 5, no. 2, pp. 189-202, 1969.

[12] J. Drury, C. Cocking, and S. Reicher, "The nature of collective resilience: Survivor reactions to the 2005 london bombings," International Journal of Mass Emergencies and Disasters, vol. 27, no. 1, pp. 66-95, 2009.

[13] J. Norris R., "Panic and the breakdown of social order: Popular myth, social theory, empirical evidence," University of Cincinnati, pp. 171183, 1987.

[14] E. L. Quarantelli, "The nature and conditions of panic," American Journal of Sociology, pp. 267-275, 1954.

[15] A. Benigno E., T. Manuel R, G. Kimberly B., and H. H Lawrence, "Normative collective behavior in the station building fire," Social Science Quarerly, pp. 100-118, 2011.

[16] J. Tsai, N. Fridman, E. Bowring, M. Brown, S. Epstein, G. Kaminka, S. Marsella, A. Ogden, I. Rika, A. Sheel, M. E. Taylor, X. Wang, A. Zilka, and M. Tambe, "Escapes: Evacuation simulation with children, authorities, parents, emotions, and social comparison," in Proc. of AAMAS'11. IFAAMAS, 2011, pp. 457-464.

[17] J. Drury and C. Cocking, "The mass psychology of disasters and emergency evacuations: A research report and implications for practice," University of Sussex, Research report, 2007.

[18] A. Ortony, G. Clore, and A. Collins, The cognitive structure of emotions. Cambridge, MA: Cambridge University Press, 1988.

[19] R. S. Lazarus, Emotion and Adaptation. Oxford University Press, 1991.

[20] K. R. Scherer, A. Schorr, and T. Johnstone, Eds., Appraisal Processes in Emotion : Theory, Methods, Research. Oxford University Press, 2001.

[21] J. Elster, Alchemies of the Mind: Rationality and the Emotions. Cambridge University Press, 1999.

[22] E. Quarantelli, "The sociology of panic," in International Encyclopedia of the Social and Behavioural Sciences, Smelser and Baltes, Eds. New York: Pergamon Press, 2001, pp. 11 020-11 023.

[23] T. Bosse, R. Duell, Z. A. Memon, J. Treur, and C. N. Van Der Wal, "Multi-agent model for mutual absorption of emotions." ECMS, vol. 2009, pp. 212-218, 2009.

[24] A. R. Damasio, "Descartes' error: Emotion, rationality and the human brain," New York: Putnam, 1994.

[25] T. Bosse, H. Mark, C. K. Michel, T. Jan, V. D. W. C. Natalie, and V. W. Arlette, "Modelling collective decision making in groups and crowds: Integrating social contagion and interacting emotions, beliefs and intentions," Article, pp. 52-84, 2013.

[26] F. Durupinar, "From audiences to mobs: Crowd simulation with psychological factors," Thesis, 2010.

[27] H. Mark, T. Jan, v. d. W. C. Natalie, and v. W. Arlette, "Modeling the interplay of emotions, beliefs and intentions within collective decision making based on insights from social neuroscience," in International Conference on Neural Information Processing. Springer, 2010, pp. 196-206.

[28] E. D. Kuligowski, R. D. Peacock, and B. L. Hoskins, A review of building evacuation models. US Department of Commerce, National Institute of Standards and Technology Gaithersburg, MD, 2005. 
[29] S. Gwynne, E. Galea, M. Owen, P. J. Lawrence, and L. Filippidis, "A review of the methodologies used in the computer simulation of evacuation from the built environment," Building and environment, vol. 34, no. 6, pp. 741-749, 1999.

[30] J. S. Lerner, Y. Li, P. Valdesolo, and K. S. Kassam, "Emotion and decision making," Psychology, vol. 66, 2015.

[31] X. Pan, "Computational modeling of human and social behaviors for emergency egress analysis," Thesis, 2006.

[32] X. Pan, C. S. Han, K. Dauber, and K. H. Law, "A multi-agent based framework for the simulation of human and social behaviors during emergency evacuations," Thesis, 2007.

[33] F. Durupinar, N. Pelechano, J. M. Allbeck, U. Gudukbay, and N. Badler, "How the ocean personality model affects the perception of crowds," Article, pp. 22-31, 2010

[34] R. F. Fahy, "Exit89: an evacuation model for high-rise buildings," Fire Safety Science, vol. 3, pp. 815-823, 1991.

[35] N. Ketchell, A. Holt, and K. Kinsella, "A technical summary of the aea egress code," Tech. Rep. 1, AEA Technology, Tech. Rep., 2002.

[36] T. M. Kisko, R. Francis, and C. Nobel, "Evacnet4 users guide," University of Florida, 1998.

[37] T. Korhonen, S. Hostikka, S. Heliövaara, and H. Ehtamo, "Fds+ evac: an agent based fire evacuation model," in Pedestrian and Evacuation Dynamics 2008. Springer, 2010, pp. 109-120.

[38] V. M. Le, C. Adam, R. Canal, B. Gaudou, T. V. Ho, and P. Taillandier, "Simulation of the emotion dynamics in a group of agents in an evacuation situation," in Principles and Practice of MAS, ser. LNCS, N. Desai, A. Liu, and M. Winikoff, Eds. Springer, 2012, vol. 7057, pp. 604-619.

[39] Y. Murakami, K. Minami, T. Kawasoe, and T. Ishida, "Multi-agent simulation for crisis management," in Knowledge Media Networking, 2002. Proceedings. IEEE Workshop on. IEEE, 2002, pp. 135-139.

[40] M. H. Nguyen, T. V. Ho, T. N. A. Nguyen, and J.-D. Zucker, "Which behavior is best in a fire evacuation: Simulation with the metro supermarket of hanoi," in Computing and Communication Technologies, Research, Innovation, and Vision for the Future (RIVF), 2012 IEEE RIVF International Conference on. IEEE, 2012, pp. 1-6.

[41] M. H. Nguyen, T. V. Ho, and J.-D. Zucker, "A simulation model for optimise the fire evacuation configuration in the metro supermarket of hanoi," in Simulated Evolution and Learning. Springer Berlin Heidelberg, 2012, pp. 470-479.

[42] V. T. Nguyen, D. Longin, T. V. Ho, and B. Gaudou, "Integration of emotion in evacuation simulation," in Information Systems for Crisis Response and Management in Mediterranean Countries, ser. Lecture Notes in Business Information Processing, C. Hanachi, F. Bénaben, and F. Charoy, Eds. Springer, 2014, vol. 196, pp. 192-205.

[43] P. Thompson, J. Wu, and E. Marchant, "Simulex 3.0: Modelling evacuation in multi-storey buildings," Fire Safety Science, vol. 5, pp. 725-736, 1997.

[44] — "Modelling evacuation in multi-storey buildings with simulex," Fire Engineers Journal, vol. 56, pp. 6-11, 1996.

[45] F. Qiu and X. Hu, "Modeling group structures in pedestrian crowd simulation," PhD, 2009.

[46] X. H. Ta, D. Longin, B. Gaudou, and T. V. Ho, "Impact of group on the evacuation process: theory and simulation," in Proceedings of the Sixth International Symposium on Information and Communication Technology. ACM, 2015, pp. 350-357.

[47] A. Grignard, P. Taillandier, B. Gaudou, D. A. Vo, N. Q. Huynh, and A. Drogoul, "Gama 1.6: Advancing the art of complex agent-based modeling and simulation," in PRIMA 2013: Principles and Practice of MAS. Springer, 2013, pp. 117-131. 